\title{
TESTING FOR INDEPENDENCE BETWEEN FUNCTIONAL TIME SERIES
}

\author{
LAJOS HORVÁTH AND GREGORY RICE
}

\begin{abstract}
Frequently econometricians are interested in verifying a relationship between two or more time series. Such analysis is typically carried out by causality and/or independence tests which have been well studied when the data is univariate or multivariate. Modern data though is increasingly of a high dimensional or functional nature for which finite dimensional methods are not suitable. In the present paper we develop methodology to check the assumption that data obtained from two functional time series are independent. Our procedure is based on the norms of empirical cross covariance operators and is asymptotically validated when the underlying populations are assumed to be in a class of weakly dependent random functions which include the functional ARMA, ARCH and GARCH processes.
\end{abstract}

\section{INTRODUCTION AND RESULTS}

A common goal of data analysis in econometrics is to determine whether or not a relationship exists between two variables which are measured over time. A determination in either way may be useful. On one hand, if a relationship is confirmed to exist between two variables then further investigation into the strength and nature of the relationship may lead to interesting insights or effective predictive models. Conversely if there is no relationship between the two variables then an entire toolbox of statistical techniques developed to analyze two samples which are independent may be used. The problem of testing for correlation between two univariate or multivariate time series has been well treated, and we discuss the relevant literature below. However, as a by product of seemingly insatiable modern data storage technology, many data of interest exhibit such large dimension that traditional multivariate techniques are not suitable. For example, tick by tick stock return data is stored hundreds of times per second, leading to thousands of observations during a single day. In such cases a pragmatic approach is to treat the data as densely observed measurements from an underlying curve, and, after using the measurements to approximate the curve, apply statistical techniques to the curves themselves. This approach is fundamental in functional data analysis, and in recent years much effort has been put forth to adapt currently available procedures in multivariate analysis to functional data. The goal of the present paper is to develop a test for independence between two functional time series.

In the context of checking for independence between two second order stationary univariate time series, Haugh (1976) proposed a testing procedure based on sample crosscorrelation estimators. His test may be considered as an adaptation of the popular

Key words and phrases. functional observations, tests for independence, weak dependence, long run covariance function, central limit theorem .

JEL Classification C12, C32

Research supported by NSF grant DMS 1305858

(C) 2015. This manuscript version is made available under the Elsevier user license http://www.elsevier.com/open-access/userlicense/1.0/ 
Box-Ljung-Pierce portmanteau test (cf. Ljung and Box (1978)) to two samples. In a similar progression the multivariate portmanteau test of Li and Mcleod (1981) was extended to test for correlation between two multivariate ARMA time series by El Himdi and Roy (1997) whose test statistic was based on cross-correlation matrices. The literature on such tests has also grown over the years to include adaptations for robustness as well as several other considerations, see Koch and Yang (1986), Li and Hui (1996) and El Himdi et al (2003) for details. Many of these results are summarized in Li (2004). A separate approach for multivariate data based on the distance correlation measure is developed in Székely and Rizzo (2013).

The analysis of functional time series has seen increased attention in statistics, economics and in other applications over the last decade, see Horváth and Kokoszka (2012) for a summary of recent advances. To test for independence within a single functional time series, Gabrys and Kokoszka (2007) proposed a method where the functional observations are projected onto finitely many basis elements, and a multivariate portmanteau test is applied to the vectors of scores which define the projections. Horváth et al (2013) developed a portmanteau test for functional data in which the inference is performed using the operator norm of the empirical covariance operators at lags $h, 1 \leq h \leq H$, which could be considered as a direct functional analog of the Box-Ljung-Pierce test. Due to the infinite dimension of functional data, a normal limit is established for the test statistic rather than the classical $\chi^{2}$ limit with degrees of freedom depending on the data dimension.

The method that we propose for testing noncorrelation between two functional time series follows the example of Horváth et al (2013). Suppose that we have observed $X_{1}(t), \ldots, X_{n}(t)$ and $Y_{1}(s), \ldots, Y_{n}(s)$ which are samples from jointly stationary sequences of square integrable random functions on $[0,1]$. Formally we are interested in testing

$$
H_{0} \text { : the sequences }\left\{X_{i}\right\}_{i=1}^{\infty} \text { and }\left\{Y_{j}\right\}_{j=1}^{\infty} \text { are independent }
$$

against the alternative

$$
\begin{aligned}
& H_{A}: \text { for some integer } h_{0},-\infty<h_{0}<\infty, \iint C_{h_{0}}^{2}(t, s) d t d s>0 \\
& \quad \text { where } C_{h_{0}}(t, s)=\operatorname{Cov}\left(X_{0}(t), Y_{h_{0}}(s)\right) .
\end{aligned}
$$

We use the notation $\int$ to mean $\int_{0}^{1}$. Assuming jointly Gaussian distributions for the underlying observations, independence reduces to zero cross-correlations at all lags, and hence $H_{A}$ is equivalent to the complement of $H_{0}$ in that case. To derive the test statistic, we note that under $H_{0}$, the sample cross-covariance functions

$$
\hat{C}_{n, h}(t, s)= \begin{cases}\frac{1}{n} \sum_{i=1}^{n-h}\left(X_{i}(t)-\bar{X}(t)\right)\left(Y_{i+h}(s)-\bar{Y}(s)\right) & h \geq 0 \\ \frac{1}{n} \sum_{i=1-h}^{n}\left(X_{i}(t)-\bar{X}(t)\right)\left(Y_{i+h}(s)-\bar{Y}(s)\right) & h<0\end{cases}
$$

should be close to the zero function for all choices of $h$, where

$$
\bar{X}(t)=\frac{1}{n} \sum_{i=1}^{n} X_{i}(t), \text { and } \bar{Y}(s)=\frac{1}{n} \sum_{i=1}^{n} Y_{i}(s) .
$$


Under $H_{A}$ a cross covariance function is different from the zero function for at least one $h$. The test statistic is then based on

$$
\hat{T}_{n, H}=\sum_{h=-H}^{H} \iint \hat{C}_{n, h}^{2}(t, s) d t d s
$$

with the hope that it includes the covariance estimator corresponding to a non zero function if it exists. We then reject $H_{0}$ for large values of $\hat{T}_{n, H}$. Our main result is the asymptotic distribution of $\hat{T}_{n, H}$ under $H_{0}$.

In order to cover a large class of functional time series processes, we assume that $\mathbf{X}=$ $\left\{X_{i}\right\}_{i=-\infty}^{\infty}$ and $\mathbf{Y}=\left\{Y_{i}\right\}_{i=-\infty}^{\infty}$ are approximable Bernoulli shifts. We say that $\boldsymbol{\eta}=$ $\left\{\eta_{j}(t)\right\}_{j=-\infty}^{\infty}$ is an $L^{4}$ absolutely approximable Bernoulli shift in $\left\{\epsilon_{j}(t),-\infty<j<\infty\right\}$ if

$$
\eta_{i}=g\left(\epsilon_{i}, \epsilon_{i-1}, \ldots\right) \text { for some nonrandom measurable function }
$$

$g: S^{\infty} \mapsto L^{2}$ and i.i.d. random innovations $\epsilon_{j},-\infty<j<\infty$,

with values in a measurable space $S$,

and

(1.3) the sequence $\{\boldsymbol{\eta}\}$ can be approximated by $\ell$-dependent sequences $\left\{\eta_{j, \ell}\right\}_{j=-\infty}^{\infty}$ in the sense that

$$
\sum_{\ell=1}^{\infty} \ell\left(E\left\|\eta_{j}-\eta_{j, \ell}\right\|^{4}\right)^{1 / 4}<\infty
$$

where $\eta_{j, \ell}$ is defined by $\eta_{j, \ell}=g\left(\epsilon_{j}, \epsilon_{j-1}, \ldots, \epsilon_{j-\ell+1}, \boldsymbol{\epsilon}_{j, \ell}^{*}\right)$,

$\boldsymbol{\epsilon}_{j, \ell}^{*}=\left(\epsilon_{j, \ell, j-\ell}^{*}, \epsilon_{j, \ell, j-\ell-1}^{*}, \ldots\right)$, where the $\epsilon_{j, \ell, k}^{*}$ 's are independent copies of

$\epsilon_{0}$, independent of $\left\{\epsilon_{j},-\infty<j<\infty\right\}$.

In assumption (1.1) we take $S$ to be an arbitrary measurable space, however in most applications $S$ is itself a function space and the evaluation of $g\left(\epsilon_{i}, \epsilon_{i-1}, \ldots\right)$ is a functional of $\left\{\epsilon_{j}(t)\right\}_{j=-\infty}^{i}$. In this case assumption (1.2) follows from the joint measurability of the $\epsilon_{i}(t, \omega)$ 's. Assumption (1.3) is stronger than the requirement $\sum_{\ell=1}^{\infty}\left(E\left\|\eta_{j}-\eta_{j, \ell}\right\|^{2}\right)^{1 / 2}<\infty$ used by Hörmann and Kokoszka (2010), Berkes et al (2013) and Jirak (2013) to establish the central limit theorem for sums of Bernoulli shifts. Since we need the central limit theorem for sample correlations, higher moment conditions and a faster rate in the approximability with $\ell$-dependent sequences are needed.

We assume that the sequences $\mathbf{X}$ and $\mathbf{Y}$ satisfy the following conditions:

Assumption 1.1. $E\left\|X_{0}\right\|^{4+\delta}<\infty$ and $E\left\|Y_{0}\right\|^{4+\delta}<\infty$ with some $\delta>0$,

Assumption 1.2. $\mathbf{X}=\left\{X_{i}(t)\right\}_{i=-\infty}^{\infty}$ is an $L^{4}$ absolutely approximable Bernoulli shift in $\left\{\epsilon_{j}(t),-\infty<j<\infty\right\}$,

and

Assumption 1.3. $\mathbf{Y}=\left\{Y_{i}(t)\right\}_{i=-\infty}^{\infty}$ is an $L^{4}$ absolutely approximable Bernoulli shift in $\left\{\bar{\epsilon}_{j}(t),-\infty<j<\infty\right\}$. 
The functions defining the Bernoulli shift sequences $\mathbf{X}$ and $\mathbf{Y}$ as in (1.1) will be denoted by $g_{\mathbf{X}}$ and $g_{\mathbf{Y}}$, respectively. The independence of the sequences $\mathbf{X}$ and $\mathbf{Y}$ stated under $H_{0}$ is conveniently given by:

Assumption 1.4. The sequences $\left\{\epsilon_{j}(t),-\infty<j<\infty\right\}$ and $\left\{\bar{\epsilon}_{j}(t),-\infty<j<\infty\right\}$ are independent.

The parameter $H$ defines the number of lags used to define the test statistic. As $n$ increases it becomes possible to accurately estimate cross covariances for larger lags, and thus we allow $H$ to tend to infinity with the sample size. Namely,

Assumption 1.5. $H=H(n) \rightarrow \infty$ and $H n^{-\tau} \rightarrow 0$, as $n \rightarrow \infty$, with some $0<\tau<$ $2 \delta /(4+7 \delta)$, where $\delta$ is defined in Assumption 1.1 .

To state the limit result for $\hat{T}_{n, H}$ we first introduce the asymptotic expected value and variance. Let for all $-\infty<j<\infty$

$$
\gamma_{\mathbf{X}}(j)=\int \operatorname{cov}\left(X_{0}(t), X_{j}(t)\right) d t, \quad \gamma_{\mathbf{Y}}(j)=\int \operatorname{cov}\left(Y_{0}(t), Y_{j}(t)\right) d t
$$

and define

$$
\mu=\sum_{j=-\infty}^{\infty} \gamma_{\mathbf{X}}(j) \gamma_{\mathbf{Y}}(j)
$$

It is shown in Lemma B.1 that under Assumptions 1.2 and 1.3 the infinite sum in the definition of $\mu$ above is absolutely convergent. Let

$$
\sigma_{h}^{2}=2 \int \cdots \int\left(\sum_{\ell=-\infty}^{\infty} \operatorname{cov}\left(X_{0}(t), X_{\ell}(s)\right) \operatorname{cov}\left(Y_{0}(u), Y_{\ell+h}(v)\right)\right)^{2} d t d s d u d v
$$

and

$$
\sigma^{2}=\sum_{h=-\infty}^{\infty} \sigma_{h}^{2}
$$

Theorem 1.1. If Assumptions 1.1-1.5 hold, then we have

$$
\frac{n \hat{T}_{n, H}-(2 H+1) \mu}{(2 H+1)^{1 / 2} \sigma} \stackrel{\mathcal{D}}{\rightarrow} \mathcal{N}
$$

where $\mathcal{N}$ stands for a standard normal random variable.

Upon the estimation of $\mu$ and $\sigma^{2}$ this result supplies an asymptotic test for $H_{0}$.

The rest of the paper is organized as follows. In Section 2 we discuss the estimation of the parameters appearing in Theorem 1.1 as well as a simulation study of how the limit result is manifested in finite sample sizes. The statistical utility of Theorem 1.1 is then illustrated by an application to tick data from several stocks listed on the NYSE. The proof of the main result is given in Section 3. The paper concludes with two appendices which contain some technical results needed in Section 3. 


\section{Finite SAMPle PROPERTIES AND AN APPLICATION}

2.1. Parameter estimates. In order to use Theorem 1.1 for data analysis it is necessary to estimate $\mu$ and $\sigma^{2}$ from the sample. Let

$$
\hat{\gamma}_{\mathbf{X}, \ell}(t, s)= \begin{cases}\frac{1}{n} \sum_{i=1}^{n-\ell}\left(X_{i}(t)-\bar{X}(t)\right)\left(X_{i+\ell}(s)-\bar{X}(s)\right) & \ell \geq 0 \\ \frac{1}{n} \sum_{i=1-\ell}^{n}\left(X_{i}(t)-\bar{X}(t)\right)\left(X_{i+\ell}(s)-\bar{X}(s)\right) & \ell<0\end{cases}
$$

and

$$
\hat{\gamma}_{\mathbf{Y}, \ell}(t, s)= \begin{cases}\frac{1}{n} \sum_{i=1}^{n-\ell}\left(Y_{i}(t)-\bar{Y}(t)\right)\left(Y_{i+\ell}(s)-\bar{Y}(s)\right) & \ell \geq 0 \\ \frac{1}{n} \sum_{i=1-\ell}^{n}\left(Y_{i}(t)-\bar{Y}(t)\right)\left(Y_{i+\ell}(s)-\bar{Y}(s)\right) & \ell<0 .\end{cases}
$$

Since $\mu$ is an infinite sum of integrated correlations, it is natural to use a kernel estimator of the form

$$
\hat{\mu}_{n}=\sum_{\ell=1-n}^{n-1} K_{1}\left(\frac{\ell}{w_{1}}\right) \int \hat{\gamma}_{\mathbf{X}, \ell}(t, t) d t \int \hat{\gamma}_{\mathbf{Y}, \ell}(s, s) d s
$$

where $K_{1}$ is a kernel function with window $w_{1}=w_{1}(n)$. According to the definition in (1.5), $\sigma^{2}$ is an infinite sum of integrals of squared correlations and so we can use again a kernel type estimator:

$$
\hat{\sigma}_{n}^{2}=\sum_{h=-2 H}^{2 H} K_{2}\left(\frac{h}{w_{2}}\right) \hat{\tau}_{n, h}
$$

where $K_{2}$ is a kernel with window $w_{2}=w_{2}(H)$,

$$
\hat{\tau}_{n, h}=2 \int \cdots \int\left(\sum_{\ell=h-n}^{n-h}\left(1-\frac{|\ell|}{n}\right) \hat{\gamma}_{\mathbf{X}, \ell}(t, s) \hat{\gamma}_{\mathbf{Y}, \ell+h}(u, v)\right)^{2} d t d s d u d v .
$$

We note that $\sigma^{2}$ is essentially the long run variance of the integrals $\iint \hat{C}_{n, h}^{2}(t, s) d t d s,-2 H \leq$ $h \leq 2 H$, and $\tau_{h}$ is the correlation of lag $h$ between these integrals. Hence $\hat{\tau}_{n, h}$ can be considered as an estimated correlation and $\hat{\sigma}_{n}^{2}$ is a kernel long run variance estimator based on the estimated correlations. We assume that the kernels $K_{i}, i=1,2$ satisfy standard regularity conditions: (i) $K_{i}(0)=1$, (ii) $K_{i}$ is continuous and bounded (iii) there is $c>0$ such that $K_{i}(u)=0$ if $|u|>c$. It can be shown if $w_{1}=w_{1}(n) \rightarrow$ $\infty, w_{1}(n) / n^{1 / 2} \rightarrow 0$ as $n \rightarrow \infty$, then $\hat{\mu}_{n}=\mu+o_{P}\left(H^{-1 / 2}\right)$ under Assumption 1.5. If $w_{2}=w_{2}(H) \rightarrow \infty, w_{2}(H) / H \rightarrow 0$, as $H \rightarrow \infty$, then one can show that $\hat{\sigma}_{n}^{2} \rightarrow \sigma^{2}$ in probability as $n \rightarrow \infty$. Hence Theorem 1.1 yields

$$
V_{n, H}=\frac{n \hat{T}_{n, H}-(2 H+1) \hat{\mu}_{n}}{(2 H+1)^{1 / 2} \hat{\sigma}_{n}} \stackrel{\mathcal{D}}{\rightarrow} \mathcal{N},
$$

where $\mathcal{N}$ stands for a standard normal random variable. 
2.2. Simulation study. We now turn to a small simulation study which compares the limit result in Theorem 1.1 to the finite sample behavior of $\hat{T}_{n, H}$. In order to describe the data generating processes (DGP's) used below, let $\left\{W_{X, i}\right\}_{i=1}^{n}$ and $\left\{W_{Y, i}\right\}_{i=1}^{n}$ be independent sequences of iid standard Brownian motions on [0,1]. Two DGP's were considered which each satisfy the conditions of Theorem 1.1:

IID: two samples of independent Brownian motions, i.e. $X_{i}(t)=W_{X, i}(t)$,

$$
Y_{i}(t)=W_{Y, i}(t), 1 \leq i \leq n, t \in[0,1]
$$

and

$\operatorname{FAR}_{q}(1)$ : two samples which follow a stationary functional autoregressive model of order one, i.e. for $1 \leq i \leq n, t \in[0,1]$,

$$
X_{i}(t)=\int \psi_{q}(t, u) X_{i-1}(u) d u+W_{X, i}(t), \quad Y_{i}(t)=\int \psi_{q}(t, u) Y_{i-1}(u) d u+W_{Y, i}(t),
$$

where $\psi_{q}(t, u)=q \min (t, u)$.

The motivation behind using Brownian motions for the error sequences in each of the DGP's is due to our application in Section 2.3 to cumulative intraday returns which often look like realizations of Brownian motions, see Figure 2.2 below. A precise but somewhat technical condition for the existence of a stationarity solution in the $\mathrm{FAR}_{q}(1)$ model is given in Bosq (2000), however it is sufficient to assume that the operator norm of $\psi$ is less than one. In our case we take $q=.75,1.5,2.25$ so that $\|\psi\| \approx .25, .5, .75$, respectively. To simulate the stationary $\operatorname{FAR}_{q}(1)$ process we used a burn in sample of size 100 starting from an independent innovation. In order to compute the statistic $V_{n, H}$ of (2.1) we must select the kernels $K_{1}$ and $K_{2}$ as well as the windows $w_{1}$ and $w_{2}$ used to compute the estimates $\hat{\mu}_{n}$ and $\hat{\sigma}_{n}^{2}$. For our analysis we used Bartlett kernels

$$
K_{1}(t)=K_{2}(t)= \begin{cases}1-|t|, & 0 \leq|t|<1 \\ 0, & |t| \geq 1\end{cases}
$$

and windows $w_{1}(r)=w_{2}(r)=\left\lceil r^{1 / 4}\right\rceil$. The simulations results below were repeated for several other common choices of kernel functions including the Parzen and flat-top kernels, as well as different choices for the window parameters. The changes in the results were negligible for different choices of the kernel functions. The results were also stable over window choices between $w_{i}(r)=\left\lceil r^{1 / 4}\right\rceil$ to $w_{i}(r)=\left\lceil r^{1 / 2}\right\rceil$ which constitute the usual range of acceptable windows. Each DGP was simulated 1000 times independently for various values of $n$ and $H$, and the percentage of test statistics $V_{n, H}$ which exceeded the 10,5 , and 1 percent critical values of the standard normal distribution are reported in Table 2.1.

Based on this data we have the following remarks:

(i) When the level of dependence within the processes is not too strong the tail of the distribution of $V_{n, H}$ appears to be approaching that of the standard normal as $n \rightarrow \infty$.

(ii) There appears to be little effect in the results due to the choice of $H$ when the dependence is weak. Even for values of $H$ close to $n^{1 / 2}$ we still observe finite sample distributions which are close to the limit. 


\begin{tabular}{|c|c|c|c|c|c|c|c|c|c|c|c|c|c|}
\hline \multicolumn{2}{|c|}{ DGP } & \multicolumn{3}{|c|}{ IID } & \multicolumn{3}{|c|}{ FAR $_{.75}(1)$} & \multicolumn{3}{|c|}{$\mathrm{FAR}_{1.5}(1)$} & \multicolumn{3}{|c|}{$\mathrm{FAR}_{2.25}(1)$} \\
\hline$N$ & $\mathrm{H}$ & $10 \%$ & $5 \%$ & $1 \%$ & $10 \%$ & $5 \%$ & $1 \%$ & $10 \%$ & $5 \%$ & $1 \%$ & $10 \%$ & $5 \%$ & $1 \%$ \\
\hline \multirow[t]{3}{*}{50} & 2 & 13.8 & 8.0 & 2.7 & 9.7 & 6.1 & 2.8 & 15.2 & 10.7 & 4.8 & 24.3 & 17.4 & 8.0 \\
\hline & 5 & 14.6 & 7.8 & 3.1 & 11.4 & 6.3 & 2.9 & 16.4 & 11.1 & 4.1 & 26.0 & 20.7 & 11.8 \\
\hline & 7 & 13.2 & 7.6 & 3.8 & 10.2 & 6.0 & 2.1 & 15.5 & 10.4 & 5.0 & 33.0 & 25.4 & 13.7 \\
\hline \multirow[t]{3}{*}{100} & 3 & 11.5 & 7.0 & 2.7 & 9.5 & 6.0 & 2.4 & 12.4 & 7.0 & 3.1 & 26.8 & 20.1 & 13.3 \\
\hline & 5 & 10.3 & 5.1 & 2.1 & 8.5 & 4.7 & 0.8 & 12.8 & 7.2 & 3.8 & 26.4 & 22.5 & 15.8 \\
\hline & 10 & 9.8 & 3.9 & 1.3 & 8.7 & 3.8 & 0.9 & 11.4 & 6.5 & 2.7 & 32.0 & 27.3 & 20.1 \\
\hline \multirow[t]{3}{*}{200} & 4 & 10.0 & 6.6 & 2.5 & 10.2 & 5.8 & 2.1 & 10.9 & 6.4 & 3.0 & 16.5 & 12.8 & 7.2 \\
\hline & 7 & 8.7 & 4.8 & 1.2 & 11.0 & 7.1 & 2.5 & 10.5 & 6.0 & 1.9 & 21.4 & 17.7 & 12.6 \\
\hline & 15 & 9.8 & 5.2 & 1.3 & 8.4 & 4.3 & 1.7 & 10.4 & 7.0 & 2.7 & 25.5 & 20.6 & 14.7 \\
\hline \multirow[t]{3}{*}{300} & 5 & 10.3 & 5.4 & 1.2 & 9.7 & 6.0 & 2.1 & 9.7 & 5.5 & 2.2 & 15.3 & 10.5 & 6.8 \\
\hline & 10 & 10.5 & 5.7 & 1.4 & 10.0 & 5.7 & 2.3 & 10.4 & 5.3 & 1.9 & 21.0 & 18.1 & 12.7 \\
\hline & 17 & 9.8 & 4.7 & 1.1 & 10.1 & 5.1 & 1.2 & 10.9 & 5.7 & 2.4 & 26.4 & 22.5 & 16.4 \\
\hline
\end{tabular}

TABLE 2.1. The percentage of 1000 simulated values of $V_{n, H}$ which exceeded the $10 \%, 5 \%$, and $1 \%$ critical values of the standard normal distribution for the DGP's IID and $\operatorname{FAR}_{q}(1)$.

(iii) When the dependence is strong, i.e. $q=2.25$, the tail of the distribution of $V_{n, H}$ becomes significantly heavier than that of the standard normal. This effect is worsened by increasing $H$.

(iv) In the context of hypothesis testing, $V_{n, H}$ achieves good size even when the temporal dependence is weak to moderate as long as the sample size is large. If strong dependence is suspected in the data then the distribution of $V_{n, H}$ may be skewed to the right.

2.3. Application to cumulative intraday stock returns. A natural example of functional data are intraday price curves constructed from densely observed price records of an asset. Five of such curves constructed from the 1 minute average stock price of Apple are shown in Figure 2.1. Several authors have studied the analysis of the shapes of price curves, see Müller et al (2011) and Kokoszka et al (2013) for a small sample of such work. In our application we use (2.1) to test for correlation between samples of curves constructed from pairs of 20 of the highest trade volume stocks listed on the New York Stock Exchange. The data we consider was obtained from www.nasdaq.com and ww. finam. com and consists of the 1 minute average stock prices of the stocks listed in Table 2.2 from January 1st, 2013 to December 11, 2013, which comprise 254 daily records per stocks. Since the test statistic is based on integrals of the sampled curves its value changes little due to the way the curves are constructed from the original observations, and thus we simply used linear interpolation. It is argued in Horváth et al (2014) that price curves themselves are typically not stationary and thus must be suitably transformed before analysis assuming stationarity may be performed. Scaler price data is typically transformed to appear closer to stationary by taking the log differences, and in our example we employ a similar technique for functional data which was proposed in Gabrys et al (2010): 
FiguRE 2.1. Five functional data objects constructed from the 1-minute average price of Apple stock. The vertical lines separate days.

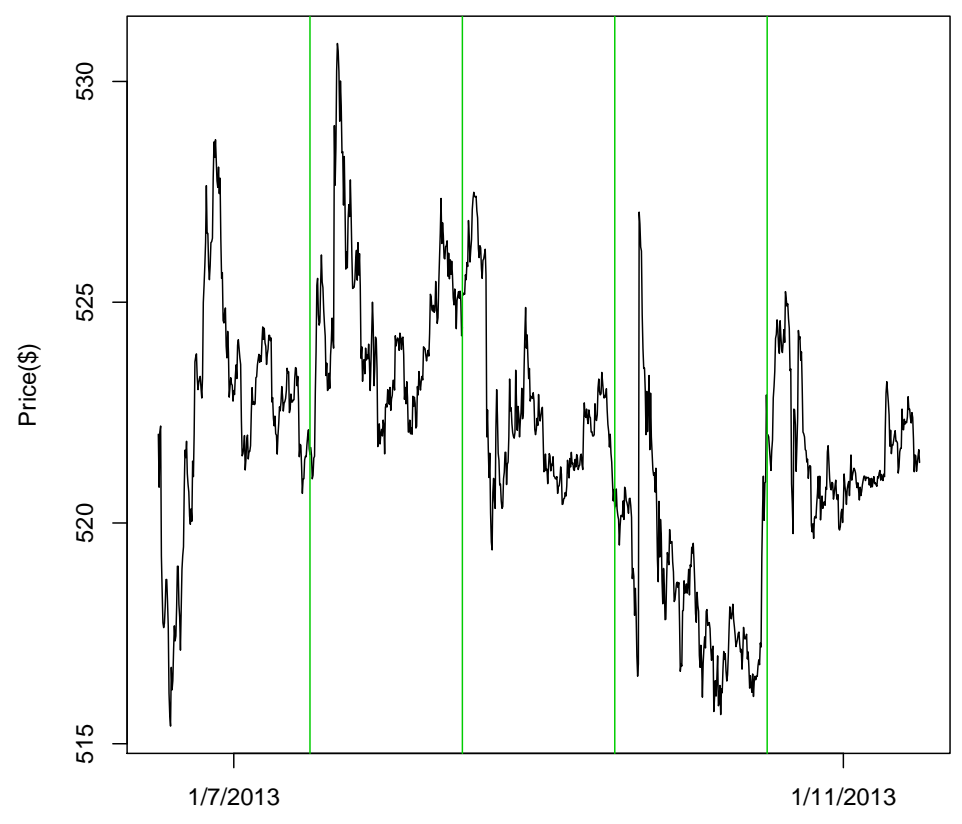

Definition 2.1. Suppose $P_{n}\left(t_{j}\right), n=1, \ldots, N, j=1, \ldots, m$, is the price of a financial asset at time $t_{j}$ on day $n$. The functions

$$
R_{n}\left(t_{j}\right)=100\left[\ln P_{n}\left(t_{j}\right)-\ln P_{n}\left(t_{1}\right)\right], \quad j=1,2, \ldots, m, \quad n=1, \ldots, N,
$$

are called the cumulative intraday returns (CIDR's).

\begin{tabular}{c|c|c|c} 
Company Name & Ticker Symbol & Company Name & Ticker Symbol \\
\hline Apple & AAPL & AT\&T & ATT \\
Bank of America & BAC & Boeing & BA \\
Chevron & CVX & Cisco Systems & CSCO \\
Citigroup & C & Coca Cola & KO \\
DuPont & DD & Exxon Mobil & XOM \\
Google & GOOG & HP & HPQ \\
IBM & IBM & Intel & INTC \\
McDonalds & MCD & Microsoft & MSFT \\
Verizon & VZ & Walmart & WMT \\
Disney & DIS & Yahoo & YHOO
\end{tabular}

TABLE 2.2. Company names and ticker symbols for the 20 stocks which were compared.

The CIDR's of the five curves in Figure 2.1 are shown in Figure 2.2. It is apparent that the overall shapes of the price curves are retained by the CIDR's and, since the CIDR's always start from zero, level stationarity is enforced. A more rigorous argument for the stationarity of the CIDR's is given in Horváth et al (2014). 
FiguRe 2.2. Five cumulative intraday return curves constructed from the price curves displayed in Figure 2.1.

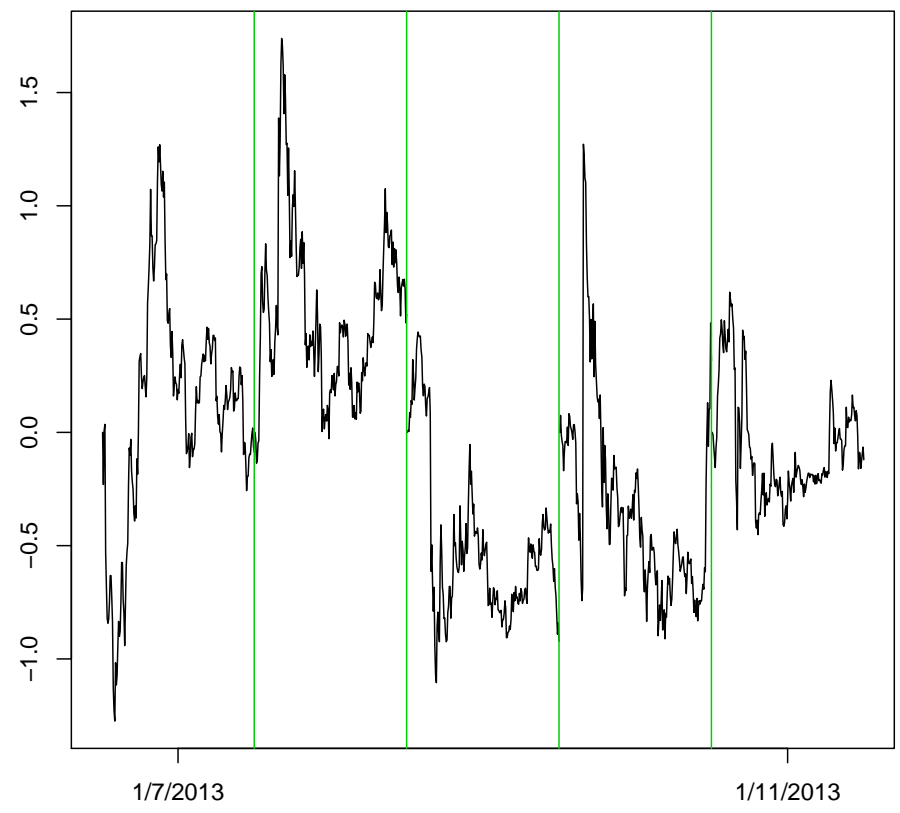

Based on the 20 stocks we used there are 190 pairs of CIDR samples for comparison. For each pair the test statistic $V_{n, H}$ was computed as outlined in Section 2.2 using $w_{1}(r)=w_{2}(r)=\left\lceil r^{1 / 4}\right\rceil$ and $H=4 \approx(254)^{1 / 4}$. An approximate $p$-value of the test was then calculated by taking $1-\Phi\left(V_{n, H}\right)$, where $\Phi$ is the standard normal distribution function. Of the $190 p$-values computed $70 \%$ were less than .05 indicating that there is strong correlation between the CIDR's of most pair of stocks listed on the NYSE. More insight can be achieved by looking at individual comparisons. Table 2.3 contains the $p$-values of all comparisons of a subset of 9 of the stocks. Some clusters are apparent, like the technology companies (AAPL,IBM,GOOG), energy companies (CVX,XOM) and service companies (WMT,DIS,MCD) for which all of the within group comparisons give effectively zero $p$-values. Also many of the comparisons between the tech companies and energy companies yielded large $p$-values, indicating that these groups of CIDR's exhibit little cross correlation. Similar clusters became apparent among the rest of the sample of 20 stocks, and using cluster analysis methods based on the values of $V_{n, H}$ could lead to further insights. For example, one could say that stocks A and B are similar if the value of $V_{n, H}$ computed between the CIDR's from A and B exceeds a certain threshold, i.e. stocks $\mathrm{A}$ and $\mathrm{B}$ are highly correlated. This will not lead to perfect clustering, however, using correlation clustering algorithms as in Kriegel et al (2009), the number of similarities across clusters or dissimilarities within clusters can be minimized.

\section{Proof of Theorem 1.1}

The proof of Theorem 1.1 requires several steps. First we claim that it is enough to prove Theorem 1.1 for the square integrals of the correlations without centering by the 


\begin{tabular}{l|ccccccccc} 
IBM & .000 & & & & & & & \\
GOOG & .001 & .000 & & & & & & \\
XOM & .541 & .612 & .051 & & & & & \\
CVX & .531 & .521 & .003 & .000 & & & & \\
C & .329 & .007 & .006 & .008 & .001 & & & \\
WMT & .602 & .358 & .000 & .001 & .000 & .000 & & \\
DIS & .221 & .002 & .000 & .014 & .000 & .000 & .001 & \\
MCD & .000 & .018 & .457 & .185 & .000 & .133 & .000 & .043 \\
\hline & AAPL & IBM & GOOG & XOM & CVX & C & WMT & DIS
\end{tabular}

TABLE 2.3. Approximate $p$-values for a test of $H_{0}$ based on $V_{n, H}$ of (2.1) for all pairwise comparisons of the 9 stocks AAPL, IBM, GOOG, XOM, CVX, C, WMT, DIS, and MCD.

sample means. In the second step we argue that $\hat{T}_{n, h}$ can be approximated with the sum of integrated squared correlations of $m$-dependent random functions if $m$ and $n$ are both large enough. The last step is the proof of the central limit theorem for the sum of integrated squared correlations of $m$-dependent functions for every fixed $m$, when $n \rightarrow \infty$. This is established using a blocking argument.

It is easy to see that $\hat{T}_{n, H}$ does not depend on the means of the observations, and so we can assume without loss of generality that

$$
E X_{0}(t)=0 \quad \text { and } \quad E Y_{0}(t)=0
$$

In order to simplify the calculations we define

$$
\tilde{C}_{n, h}(t, s)=\frac{1}{n} \sum_{i=1}^{n} X_{i}(t) Y_{i+h}(s), \quad-H \leq h \leq H
$$

and

$$
\tilde{T}_{n, H}=\sum_{h=-H}^{H} \tilde{\xi}_{n, h} \quad \text { with } \quad \tilde{\xi}_{n, h}=\iint \tilde{C}_{n, h}^{2}(t, s) d s .
$$

Lemma 3.1. If Assumptions 1.1-1.5 and (3.1) hold, then we have

$$
E\left[n\left(\hat{T}_{n, H}-\tilde{T}_{n, H}\right)\right]^{2}=o(H) \quad \text { as } n \rightarrow \infty .
$$

Proof. This claim can be proven by standard arguments so the details are omitted.

For every $m \geq 1$ we define according to Assumption 1.2

$$
X_{i, m}=g_{\mathbf{X}}\left(\epsilon_{i}, \epsilon_{i-1}, \ldots, \epsilon_{i-m+1}, \boldsymbol{\epsilon}_{i, m}^{*}\right)
$$

with $\boldsymbol{\epsilon}_{i, m}^{*}=\left(\epsilon_{i, m, i-m}^{*}, \epsilon_{i, m, i-m-1}^{*}, \ldots\right)$, where the $\epsilon_{j, \ell, k}^{*}$ ' are independent copies of $\epsilon_{0}$ and independent of $\left\{\epsilon_{j}, \bar{\epsilon}_{j},-\infty<j<\infty\right\}$. The random function $Y_{i, m}$ is defined analogously by

$$
Y_{i, m}=g_{\mathbf{Y}}\left(\bar{\epsilon}_{i}, \bar{\epsilon}_{i-1}, \ldots, \bar{\epsilon}_{i-m+1}, \overline{\boldsymbol{\epsilon}}_{i, m}^{*}\right),
$$

with $\overline{\boldsymbol{\epsilon}}_{i, m}^{*}=\left(\bar{\epsilon}_{i, m, i-m}^{*}, \bar{\epsilon}_{i, m, i-m-1}^{*}, \ldots\right)$, where the $\bar{\epsilon}_{j, \ell, k}^{*}$ ' are independent copies of $\bar{\epsilon}_{0}$ and independent of $\left\{\epsilon_{j}, \bar{\epsilon}_{j}, \epsilon_{j, \ell, k}^{*},-\infty<j, k, \ell<\infty\right\}$. It follows from the definition that both $X_{i, m},-\infty<i<\infty$ and $Y_{i, m},-\infty<i<\infty$ are stationary $m$-dependent sequences, 
independent of each other. Also, for every $i$ and $m$ we have that $X_{i, m}=X_{0}$ and $Y_{i, m}=Y_{0}$ in distribution. Next we introduce

$$
\bar{C}_{m}(t, s)=\bar{C}_{n, h, m}(t, s)=\frac{1}{n} \sum_{i=1}^{n} X_{i, m}(t) Y_{i+h, m}(s), \quad-H \leq h \leq H
$$

and

$$
\bar{T}_{n, H, m}=\sum_{h=-H}^{H} \bar{\xi}_{n, h, m} \quad \text { with } \quad \bar{\xi}_{n, h, m}=\iint \bar{C}_{n, h, m}^{2}(t, s) d s .
$$

We also use the notation

$$
J_{\mathbf{X}}(h)=\sum_{i=h}^{\infty}\left(E\left\|X_{0}-X_{0, i}\right\|^{4}\right)^{1 / 4}, \quad G_{\mathbf{X}}(h)=\sum_{i=h}^{\infty} i\left(E\left\|X_{0}-X_{0, i}\right\|^{2}\right)^{1 / 2}
$$

and similarly

$$
J_{\mathbf{Y}}(h)=\sum_{i=h}^{\infty}\left(E\left\|Y_{0}-Y_{0, i}\right\|^{4}\right)^{1 / 4}, \quad G_{\mathbf{Y}}(h)=\sum_{i=h}^{\infty} i\left(E\left\|Y_{0}-Y_{0, i}\right\|^{2}\right)^{1 / 2} .
$$

in the rest of the proofs.

Lemma 3.2. If Assumptions 1.1-1.5 and (3.1) hold, then we have

$$
\lim _{m \rightarrow \infty} \limsup _{n \rightarrow \infty} E\left[\frac{n}{H^{1 / 2}}\left\{\left(\tilde{T}_{n, H}-E \tilde{T}_{n, H}\right)-\left(\bar{T}_{n, H, m}-E \bar{T}_{n, H, m}\right)\right\}\right]^{2}=0 .
$$

Proof. By the stationarity of $\left\{\left(X_{i}, X_{i, m}\right),-\infty<i<\infty\right\}$ and $\left\{\left(Y_{i}, Y_{i, m}\right),-\infty<i<\infty\right\}$ we have

$$
\begin{aligned}
& E\left\{\left(\tilde{T}_{n, H}-E \tilde{T}_{n, H}\right)-\left(\bar{T}_{n, H, m}-E \bar{T}_{n, H, m}\right)\right\}^{2} \\
& \quad \leq(2 H+1) \operatorname{var}\left(\tilde{\xi}_{n, 0}-\bar{\xi}_{n, 0, m}\right)+4 H \sum_{h=1}^{2 H}\left|\operatorname{cov}\left(\tilde{\xi}_{n, 0}-\bar{\xi}_{n, 0, m}, \tilde{\xi}_{n, h}-\bar{\xi}_{n, h, m}\right)\right| .
\end{aligned}
$$

It follows from Assumptions 1.2-1.4 that for every fixed $h$

$$
\lim _{m \rightarrow \infty} \limsup _{n \rightarrow \infty} n^{2} \operatorname{cov}\left(\tilde{\xi}_{n, 0}-\bar{\xi}_{n, 0, m}, \tilde{\xi}_{n, h}-\bar{\xi}_{n, h, m}\right)=0 .
$$

For any $M \geq 1$ we write

$$
\begin{aligned}
& \sum_{h=1}^{2 H}\left|\operatorname{cov}\left(\tilde{\xi}_{n, 0}-\bar{\xi}_{n, 0, m}, \tilde{\xi}_{n, h}-\bar{\xi}_{n, h, m}\right)\right| \\
& \leq \sum_{h=1}^{M}\left|\operatorname{cov}\left(\tilde{\xi}_{n, 0}-\bar{\xi}_{n, 0, m}, \tilde{\xi}_{n, h}-\bar{\xi}_{n, h, m}\right)\right| \\
& \quad+\sum_{h=M+1}^{H}\left(\left|\operatorname{cov}\left(\tilde{\xi}_{n, 0}, \tilde{\xi}_{n, h}\right)\right|+\left|\operatorname{cov}\left(\bar{\xi}_{n, 0, m}, \bar{\xi}_{n, h, m}\right)\right|+2\left|\operatorname{cov}\left(\tilde{\xi}_{n, 0}, \bar{\xi}_{n, h, m}\right)\right|\right) .
\end{aligned}
$$


It follows from (3.4) that for all $M \geq 1$

$$
\lim _{m \rightarrow \infty} \limsup _{n \rightarrow \infty} n^{2} \sum_{h=1}^{M}\left|\operatorname{cov}\left(\tilde{\xi}_{n, 0}-\bar{\xi}_{n, 0, m}, \tilde{\xi}_{n, h}-\bar{\xi}_{n, h, m}\right)\right|=0 .
$$

We prove in Appendix A that there is a constant $C$ depending only on $E\left\|X_{0}\right\|^{4}, E\left\|Y_{0}\right\|^{4}, J_{\mathbf{X}}(0)$, $J_{\mathbf{Y}}(0), G_{\mathbf{X}}(0)$ and $G_{\mathbf{Y}}(0)$ such that for all $h \geq 1$

$$
n^{2}\left|\operatorname{cov}\left(\tilde{\xi}_{n, 0}, \tilde{\xi}_{n, h}\right)\right| \leq C\left(J_{\mathbf{X}}(h)+J_{\mathbf{Y}}(h)+\frac{h^{2}}{n}\right) .
$$

Hence we get that

$$
n^{2} \sum_{h=M+1}^{H}\left|\operatorname{cov}\left(\tilde{\xi}_{n, 0}, \tilde{\xi}_{n, h}\right)\right| \leq C\left(\sum_{h=M+1}^{\infty}\left(J_{\mathbf{X}}(h)+J_{\mathbf{Y}}(h)\right)+\frac{H^{3}}{n}\right)
$$

and therefore by Assumptions 1.2, 1.3 and 1.5

$$
\lim _{M \rightarrow \infty} \limsup _{n \rightarrow \infty} n^{2} \sum_{h=M+1}^{H}\left|\operatorname{cov}\left(\tilde{\xi}_{n, 0}, \tilde{\xi}_{n, h}\right)\right|=0 .
$$

Following the proof of Lemma A.1 one can verify that

$$
n^{2}\left|\operatorname{cov}\left(\tilde{\xi}_{n, 0}, \bar{\xi}_{n, h, m}\right)\right| \leq C\left(J_{\mathbf{X}}(h)+J_{\mathbf{Y}}(h)+\frac{h^{2}}{n}\right)
$$

and

$$
n^{2}\left|\operatorname{cov}\left(\bar{\xi}_{n, 0, m}, \bar{\xi}_{n, h, m}\right)\right| \leq C\left(J_{\mathbf{X}}(h)+J_{\mathbf{Y}}(h)+\frac{h^{2}}{n}\right)
$$

with the the same $C$ as in (3.6). We wish to note that the proofs of (3.9) and (3.10) are simpler than that of (3.6) due to the $m$-dependence of $X_{i, m}$ and $Y_{j, m}$. Using (3.9) and (3.10) we get that

$$
\lim _{M \rightarrow \infty} \limsup _{n \rightarrow \infty} n^{2} \sum_{h=M+1}^{H}\left(\left|\operatorname{cov}\left(\bar{\xi}_{n, 0, m}, \bar{\xi}_{n, h, m}\right)\right|+2\left|\operatorname{cov}\left(\tilde{\xi}_{n, 0}, \bar{\xi}_{n, h, m}\right)\right|\right)=0
$$

and therefore Lemma 3.2 follows from (3.5) and (3.6).

According to Lemma 3.2, it is enough to prove Theorem 1.1 for $m$ dependent variables.

Lemma 3.3. If Assumptions 1.1-1.5 and (3.1) hold, then for every $m \geq 1$ we have that

$$
\frac{n \bar{T}_{n, H, m}-(2 H+1) \bar{\mu}}{(2 H+1)^{1 / 2} \bar{\sigma}} \stackrel{\mathcal{D}}{\rightarrow} \mathcal{N}
$$

where $\mathcal{N}$ denotes a standard normal random variable.

Proof. First we define $N=H^{3} \log H$, and the sets $B(k, \ell)=\{(i, j):(k-1)(N+3 H)+1 \leq$ $i \leq k N+3(k-1) H,(\ell-1)(N+3 H)+1 \leq j \leq N+3(\ell-1) H\}, 1 \leq k, \ell \leq M$, where $M$ is defined as the largest integer satisfying $M N+3(M-1) H \leq n$, i.e. $M=\lceil n /(N+3 H)\rceil$. Let

$$
\bar{\eta}_{i, j}=\int X_{i, m}(t) X_{j, m}(t) d t \quad \text { and } \quad \bar{\theta}_{i, j}=\int Y_{i, m}(t) Y_{j, m}(t) d t
$$


and define

$$
e_{k, \ell}=\sum_{(i, j) \in B(k, \ell)} \sum_{h=-H}^{H} \bar{\eta}_{i, j} \bar{\theta}_{i+h, j+h} .
$$

Since the random variables $\bar{\eta}_{i, j}, \bar{\theta}_{i, j}$ are constructed from $m$-dependent random functions we can assume by letting $H>m \geq 1$, that $e_{k, \ell}, 1 \leq k, \ell \leq M$ are independent and identically distributed. Using Petrov (1995, p. 58) we get that

$$
E\left|e_{k, \ell}-E e_{k, \ell}\right|^{2+\delta / 2} \leq(2 H+1)^{1+\delta / 2} \sum_{h=-H}^{H} E\left|\sum_{(i, j) \in B(k, \ell)}\left[\bar{\eta}_{i, j} \bar{\theta}_{i+h, j+h}-E \bar{\eta}_{i, j} \bar{\theta}_{i+h, j+h}\right]\right|^{2+\delta / 2} .
$$

Using the stationarity and the $m$-dependence of the $X_{i, m}$ 's and the $Y_{j, m}$ 's we obtain that

$$
E\left|\sum_{(i, j) \in B(k, \ell)}\left[\bar{\eta}_{i, j} \bar{\theta}_{i+h, j+h}-E \bar{\eta}_{i, j} \bar{\theta}_{i+h, j+h}\right]\right|^{2+\delta / 2} \leq C N^{2+\delta / 2},
$$

where $C$ only depends on $m, E\left\|X_{0}\right\|^{4+\delta}$ and $E\left\|Y_{0}\right\|^{4+\delta}$. Also, it follows from Appendix B that there is $c_{0}>0$ such that

$$
\operatorname{var}\left(e_{k, \ell}\right) \geq c_{0} N^{2} H
$$

since $H^{3} / N \rightarrow 0$. Thus by Assumption 1.5 we get that

$$
\frac{\left(\sum_{1 \leq k, \ell \leq M} E\left|e_{k, \ell}-E e_{k, \ell}\right|^{2+\delta / 2}\right)^{1 /(2+\delta / 2)}}{\left(\sum_{1 \leq k, \ell \leq M} \operatorname{var}\left(e_{k, \ell}\right)\right)^{1 / 2}}=O\left(\frac{M^{2 /(2+\delta / 2)} H N}{M N H^{1 / 2}}\right)=o(1),
$$

and so by Lyapunov's theorem (cf. Petrov (1995, p. 126)) we conclude

$$
\frac{\sum_{1 \leq k, \ell \leq M}\left(e_{k, \ell}-E e_{k, \ell}\right)}{M \operatorname{var}\left(e_{1,1}\right)^{1 / 2}} \stackrel{\mathcal{D}}{\rightarrow} \mathcal{N},
$$

where $\mathcal{N}$ denotes a standard normal random variable.

Next we define the small blocks which do not contribute to the limit. Let $C(k, \ell)=$ $\{(i, j): k N+3(k-1) H+1 \leq i \leq k N+3 k H, \ell N+3(\ell-1) H+1 \leq j \leq \ell N+3 \ell H\}, 1 \leq$ $k, \ell \leq M-1$. Using Petrov (1995, p. 58) and stationarity we conclude that

$$
\operatorname{var}\left(\sum_{(i, j) \in C(k, \ell)} \sum_{h=-H}^{H} \bar{\eta}_{i, j} \bar{\theta}_{i+h, j+h}\right) \leq C_{1} H^{2} \operatorname{var}\left(\sum_{(i, j) \in C(k, \ell)} \bar{\eta}_{i, j} \bar{\theta}_{i, j}\right) \leq C_{2} H^{4}
$$

with some constants $C_{1}$ and $C_{2}$. Since $H>m$, by independence we obtain that

$$
\begin{aligned}
\operatorname{var}\left(\sum_{1 \leq k, \ell<M} \sum_{(i, j) \in C(k, \ell)} \sum_{h=-H}^{H} \bar{\eta}_{i, j} \bar{\theta}_{i+h, j+h}\right) & =O\left(M^{2} H^{4}\right) \\
& =O\left(n^{2} N^{-2} H^{4}\right)=o\left(n^{2} H\right)
\end{aligned}
$$

on account of $H^{3 / 2} / N \rightarrow 0$. Let $D_{1}(k, \ell)=\{(i, j):(k-1)(N+3 H)+1 \leq i \leq$ $k N+3(k-1) H, \ell N+3(\ell-1) H+1 \leq j \leq \ell N+3 \ell H\}, 1 \leq k \leq M, 1 \leq \ell \leq M-1$. We can divide $D_{1}(k, \ell)$ into $\lceil N / H\rceil$ blocks of size $3 H \times 3 H$ and one additional smaller 
block. The sums of $\sum_{h=-H}^{H} \bar{\eta}_{i, j} \bar{\theta}_{i+h, j+h}$ over these blocks give 2 dependent variables so by (3.11) we get that

$$
\operatorname{var}\left(\sum_{(i, j) \in D_{1}(k, \ell)} \sum_{h=-H}^{H} \bar{\eta}_{i, j} \bar{\theta}_{i+h, j+h}\right)=O\left(\frac{N}{H} H^{4}\right) .
$$

Thus we have

$$
\begin{aligned}
& \sum_{1 \leq k, \ell<M} \sum_{(i, j) \in D_{1}(k, \ell)} \sum_{h=-H}^{H}\left(\bar{\eta}_{i, j} \bar{\theta}_{i+h, j+h}-E \bar{\eta}_{i, j} \bar{\theta}_{i+h, j+h}\right) \\
& =O_{P}\left(M N^{1 / 2} H^{3 / 2}\right)=O_{P}\left(n N^{-1 / 2} H^{2}\right)=o_{P}\left(n H^{1 / 2}\right)
\end{aligned}
$$

on account of $H^{3} / N \rightarrow 0$. The same arguments give

$$
\sum_{1 \leq k, \ell<M} \sum_{(i, j) \in D_{2}(k, \ell)} \sum_{h=-H}^{H}\left(\bar{\eta}_{i, j} \bar{\theta}_{i+h, j+h}-E \bar{\eta}_{i, j} \bar{\theta}_{i+h, j+h}\right)=o_{P}\left(n H^{1 / 2}\right),
$$

where $D_{2}(k, \ell)=\{(i, j): k N+3(k-1) H+1 \leq i \leq N+3 k H,(\ell-1)(N+3 H)+$ $1 \leq j \leq \ell N+3(\ell-1) H\}, 1 \leq k \leq M-1,1 \leq \ell \leq M$. Let $D=\{1 \leq i, j \leq$ $n\} \backslash\left(\cup_{1 \leq k, \ell \leq M} C_{k, \ell} \cup_{1 \leq k \leq M, 1 \leq \ell \leq M-1} D_{1}(k, \ell) \cup_{1 \leq k \leq M-1,1 \leq \ell \leq M} D_{2}(k, \ell) \cup_{1 \leq k, \ell \leq M} B(k, \ell)\right)$. Repeating the proofs of (3.12) and (3.13) one can verify that

$$
\sum_{(i, j) \in D} \sum_{h=-H}^{H}\left(\bar{\eta}_{i, j} \bar{\theta}_{i+h, j+h}-E \bar{\eta}_{i, j} \bar{\theta}_{i+h, j+h}\right)=o_{P}\left(n H^{1 / 2}\right),
$$

completing the proof of Lemma 3.3.

Summary: The problem of testing for independence between two finite dimensional time series has been studied extensively in the literature and most available methods are based on cross covariance estimators. In this paper we established the asymptotic normality of a test statistic constructed from the operator norms of empirical cross covariance operators using functional data. This asymptotic result supplies a test of the null hypothesis that two functional time series are independent. The rate at which the asymptotic result is achieved as the sample size increases was investigated using a Monte Carlo simulation study and the results showed that the limit approximation was accurate for moderate to large sample sizes. Finally we illustrated an application of our limit result to testing for independence between cumulative intraday return curves constructed from the closing prices of stocks.

ACKNowledgements: We are grateful to two anonymous referees for their careful reading of our paper and for their helpful insights and remarks.

\section{REFERENCES}

[1] Berkes, I., Horváth, L. and Rice, G.: Weak invariance principles for sums of dependent random functions. Stochastic Processes and their Applications 123(2013), 385-403.

[2] Bosq, D.: Linear Processes in Function Spaces. Springer, New York, 2010.

[3] El Himdi, K. and Roy, R.: Tests for noncorrelation of two multivariate ARMA time series. The Canadian Journal of Statistics, 25(197), 233-256. 
[4] El Himdi, K., Roy, R. and Duchesne, P.: Tests for non-correlation of two multivariate time series: a nonparametric approach. Mathematical Statistics and Applications: Festschrift for Constance van Eeden, 397-416, IMS Lecture Notes Monogr. Ser., 42, Inst. Math. Statist., Beachwood, OH, 2003.

[5] Gabrys, R., Hörmann, S. and Kokoszka, P.: Monitoring the intraday volatility pattern. Journal of Time Series Econometrics 5(2013), 87-116.

[6] Gabrys, R. and Kokoszka, P.: Portmanteau test of independence for functional observations. Journal of the American Statistical Association 102(2007), 1338-1348.

[7] Haugh, L.D.: Checking the independence of two covariance-stationary time series: a univariate residual cross-correlation approach. Journal of the American Statistical Association, 7(1976), 378385.

[8] Hörmann, S. and Kokoszka, P.: Weakly dependent functional data. Annals of Statistics 38(2010), $1845-1884$.

[9] Horváth, L., Hušková, M. and Rice, G.: Test of independence for functional data. Journal of Multivariate Analysis, 117(2013), 100-119.

[10] Horváth, L. and Kokoszka, P.: Inference for Functional Data with Applications. Springer, New York, 2012.

[11] Horváth, L, Kokoszka, P. and Rice, G.: Testing stationarity of functional time series. Journal of Econometrics (forthcoming), 2014.

[12] Jirak, M.: On weak invariance principles for sums of dependent random functionals. Statistics 8 Probability Letters 83(2013), 2291-2296

[13] Koch, P.D. and Yang, S.: A method for testing the independence of two time series that accounts for a potential pattern in the cross-correlation function. Journal of the American Statistical Association, 81(1986), 533-544.

[14] Kokoszka, P., Miao, H. and Zhang, X.: 2013. Functional multifactor regression for intraday price curves. Technical report. Colorado State University, 2013.

[15] Kriegel, H.-S., Kröger, P. and Zimek, A.: Clustering high-dimensional data: a survey on subspace clustering, and correlation clustering. ACT Transactions on Knowledge Discovery from Data 3(2009), 1.1-1.58.

[16] Li, W.K.: Diagnostic Checks in Time Series. Chapman and Hall/CRC, New York, 2004.

[17] Li, W.K. and Hui, Y.Y.: Robust residual cross correlation tests for lagged relations in time series. Journal of Statistical Computations and Simulations. 49(1994), 103-109.

[18] Li, W.K. and McLeod, A.I.: Distribution of the residual autocorrelations in multivariate ARMA time series models. J. Royal Statistical Society, Ser. B 43(1981), 231-239.

[19] Ljung, G. and Box, G.: On a measure of lack of fit in time series models. Biometrika 66(1978), $67-72$.

[20] Müller, H-G., Sen, R. and Stadtmüller, U.: 2011. Functional data analysis for volatility. Journal of Econometrics 165(2011), 233245.

[21] Petrov, V.V.: Limit Theorems of Probability Theory. Clarendon Press, Oxford, 1995.

[22] Székely, G. and Rizzo, M.: The distance correlation t-test of independence in high dimension. Journal of Multivariate Analysis, 117(2013), 193-213.

\section{Appendix A. Bound for covariances}

Let

$$
\eta_{\ell, k}=\int X_{\ell}(t) X_{k}(t) d t, \quad \theta_{\ell, k}=\int Y_{\ell}(t) Y_{k}(t) d t
$$

and

$$
a(\ell-k)=E \eta_{\ell, k}, \quad b(\ell-k)=E \theta_{\ell, k} .
$$

Clearly, $a(x)=a(-x)$ and $b(x)=b(-x)$. Let

$$
V_{n}(h)=\operatorname{cov}\left(\sum_{i=1}^{n} \sum_{j=1}^{n} \eta_{i, j} \theta_{i, j}, \sum_{k=1}^{n} \sum_{\ell=1}^{n} \eta_{k, \ell} \theta_{k+h, \ell+h}\right) .
$$


In addition to $J_{\mathbf{X}}, J_{\mathbf{Y}}, G_{\mathbf{X}}$ and $G_{\mathbf{Y}}$ defined in Section 3 we also use

$$
L_{\mathbf{X}}(h)=\sum_{i=h}^{\infty}\left(E\left\|X_{0}-X_{0, i}\right\|^{2}\right)^{1 / 2} \text { and } L_{\mathbf{Y}}(h)=\sum_{i=h}^{\infty}\left(E\left\|Y_{0}-Y_{0, i}\right\|^{2}\right)^{1 / 2} .
$$

Lemma A.1. If Assumptions 1.1-1.5 and (3.1) hold, then for all $-H \leq h \leq H$ we have

$$
\begin{aligned}
\left|V_{n}(h)\right| \leq C & \left\{n^{2}\left(J_{\mathbf{X}}(h) J_{\mathbf{Y}}(0)+J_{\mathbf{X}}(0) J_{\mathbf{Y}}(h)\right)+n\left(J_{\mathbf{X}}(0) J_{\mathbf{Y}}(0)+G_{\mathbf{X}}(0) J_{\mathbf{Y}}(0)+J_{\mathbf{X}}(0) G_{\mathbf{Y}}(0)\right)\right. \\
& \left.\left.+n h^{2}\left(J_{\mathbf{X}}(0)+J_{\mathbf{Y}}(0)\right)+J_{\mathbf{X}}(0) J_{\mathbf{Y}}(0)\right)\right\},
\end{aligned}
$$

where $C$ only depends on $E\left\|X_{0}\right\|^{4}$ and $E\left\|Y_{0}\right\|^{4}$.

Proof. It is easy to see that by Assumptions 1.2-1.4 we have

$$
\begin{aligned}
\left|V_{n, h}\right| & =\left|\sum_{i=1}^{n} \sum_{j=1}^{n} \sum_{k=1}^{n} \sum_{\ell=1}^{n}\left(E \eta_{i, j} \eta_{k, \ell} E \theta_{i, j} \theta_{k+h, \ell+h}-a(i-j) a(k-\ell) b(i-j) b(k-\ell)\right)\right| \\
& \leq n \sum_{k=1}^{n} \sum_{\ell=1}^{n}\left|E \eta_{0,0} \eta_{k, \ell} E \theta_{0,0} \theta_{k+h, \ell+h}-a(0) a(k-\ell) b(0) b(k-\ell)\right| \\
& +2 n \sum_{j=1}^{n} \sum_{k=1}^{n} \sum_{\ell=1}^{n}\left|E \eta_{0, j} \eta_{k, \ell} E \theta_{0, j} \theta_{k+h, \ell+h}-a(j) a(k-\ell) b(j) k(k-\ell)\right| \\
& \leq 2 n \sum_{j=0}^{n} \sum_{k=1}^{n} \sum_{\ell=1}^{n}\left|E \eta_{0, j} \eta_{k, \ell} E \theta_{0, j} \theta_{k+h, \ell+h}-a(j) a(k-\ell) b(j) k(k-\ell)\right| .
\end{aligned}
$$

We proceed by bounding the sums in $j, k, \ell$ over several subsets of the indices whose union covers all possible combinations.

Case I. Let $R_{1}=\{(j, k, \ell): 0 \leq j \leq k \leq \ell \leq n\}, R_{1,1}=R_{1,1}(h)=\left\{(j, k, \ell) \in R_{1}: j \geq h\right\}$, $R_{1,2}=R_{1,2}(h)=\left\{(j, k, \ell) \in R_{1}: \ell-k \geq h\right\}$ and $R_{1,3}=R_{1,3}(h)=R_{1} \backslash\left(R_{1,1}(h) \cup R_{1,2}(h)\right)$. Using Assumption 1.2 and the Cauchy-Schwarz inequality we get for all $j \geq 0$

$$
\begin{aligned}
|a(j)| & =\left|E \int X_{0}(t) X_{j}(t) d t\right| \\
& =\mid \int E X_{0}(t)\left(\left(X_{j}(t)-X_{j, j}(t)\right) d t \mid\right. \\
& \leq \int\left(E X_{0}^{2}(t)\right)^{1 / 2}\left(E\left(X_{j}(t)-X_{j, j}(t)\right)\right)^{1 / 2} d t \\
& \leq\left(\int E X_{0}^{2}(t) d t \int E\left(X_{j}(t)-X_{j, j}(t)\right)^{2} d t\right)^{1 / 2} \\
& =\left(E\left\|X_{j}\right\|^{2}\right)^{1 / 2}\left(E\left\|X_{j}-X_{j, j}\right\|^{2}\right)^{1 / 2} \\
& =\left(E\left\|X_{0}\right\|^{2}\right)^{1 / 2}\left(E\left\|X_{0}-X_{0, j}\right\|^{2}\right)^{1 / 2},
\end{aligned}
$$

since by construction $X_{0}$ and $X_{j, j}$ are independent with zero mean. Similarly, for all $j \geq 0$ we have

$$
|b(j)| \leq\left(E\left\|Y_{0}\right\|^{2}\right)^{1 / 2}\left(E\left\|Y_{0}-Y_{0, j}\right\|^{2}\right)^{1 / 2}
$$


By bounds in (A.1) and (A.2) we conclude

$$
\frac{1}{n} \sum_{(j, k, \ell) \in R_{1,1}}|a(j) a(\ell-k) b(j) b(\ell-k)| \leq 4\left(E\left\|X_{0}\right\|^{2} E\left\|Y_{0}\right\|^{2}\right)^{3 / 2} L_{\mathbf{X}}(h) L_{\mathbf{Y}}(0) .
$$

Assumption 1.2 implies

$$
\begin{aligned}
E \eta_{0, j} \eta_{k, \ell}= & \iint E X_{0}(t) X_{j}(t) X_{k}(s) X_{\ell}(s) d t d s \\
= & \iint E X_{0}(t)\left(X_{j}(t) X_{k}(s) X_{\ell}(s)-X_{j, j}(t) X_{k, j}(s) X_{\ell, j}(s)\right) d t d s \\
= & E \int X_{0}(t)\left(X_{j}(t)-X_{j, j}(t)\right) d t \int X_{k}(s) X_{\ell}(s) d s \\
& +E \int X_{0}(t) X_{j, j}(t) d t \int X_{k}(s)\left(X_{\ell}(s)-X_{\ell, j}(s)\right) d s \\
& +E \int X_{0}(t) X_{j, j}(t) d t \int X_{\ell, j}(s)\left(X_{k}(s)-X_{k, j}(s)\right) d s .
\end{aligned}
$$

The Cauchy-Schwarz inequality and the stationarity of the $X_{\ell}$ 's gives as in (A.1) that

$$
\left|E \int X_{0}(t)\left(X_{j}(t)-X_{j, j}(t)\right) d t \int X_{k}(s) X_{\ell}(s) d s\right| \leq\left(E\left\|X_{0}\right\|^{4}\right)^{3 / 4}\left(E\left\|X_{0}-X_{0, j}\right\|^{4}\right)^{1 / 4}
$$

and similarly

$$
\begin{aligned}
& \left|E \int X_{0}(t) X_{j, j}(t) d t \int X_{k}(s)\left(X_{\ell}(s)-X_{\ell, j}(s)\right) d s\right| \leq\left(E\left\|X_{0}\right\|^{4}\right)^{3 / 4}\left(E\left\|X_{0}-X_{0, j}\right\|^{4}\right)^{1 / 4}, \\
& \left|E \int X_{0}(t) X_{j, j}(t) d t \int X_{\ell, j}(s)\left(X_{k}(s)-X_{k, j}(s)\right) d s\right| \leq\left(E\left\|X_{0}\right\|^{4}\right)^{3 / 4}\left(E\left\|X_{0}-X_{0, j}\right\|^{4}\right)^{1 / 4} .
\end{aligned}
$$

Thus we get

$$
\left|E \eta_{0, j} \eta_{k, \ell}\right| \leq 3\left(E\left\|X_{0}\right\|^{4}\right)^{3 / 4}\left(E\left\|X_{0}-X_{0, j}\right\|^{4}\right)^{1 / 4}
$$

Repeating the arguments above, one can easily verify that

$$
\left|E \theta_{0, j} \theta_{k+h, \ell+h}\right| \leq 3\left(E\left\|Y_{0}\right\|^{4}\right)^{3 / 4}\left(E\left\|Y_{0}-Y_{0, \ell-k}\right\|^{4}\right)^{1 / 4} .
$$

Combining (A.4) and (A.5) we get

$$
\sum_{(j, k, \ell) \in R_{1,1}}\left|E \eta_{0, j} \eta_{k, \ell} E \theta_{0, j} \theta_{k+h, \ell+h}\right| \leq 9 n\left(E\left\|X_{0}\right\|^{4}\right)^{3 / 4}\left(E\left\|Y_{0}\right\|^{4}\right)^{3 / 4} J_{\mathbf{X}}(h) J_{\mathbf{Y}}(0) .
$$

Minor modifications of the proofs of (A.3) and (A.6) lead to

$$
\sum_{(j, k, \ell) \in R_{1,2}}|a(j) a(\ell-k) b(j) b(\ell-k)| \leq n E\left\|X_{0}\right\|^{2} E\left\|Y_{0}\right\|^{2} L_{\mathbf{X}}(0) L_{\mathbf{Y}}(h)
$$

and

$$
\sum_{(j, k, \ell) \in R_{1,2}}\left|E \eta_{0, j} \eta_{k, \ell} E \theta_{0, j} \theta_{k+h, \ell+h}\right| \leq 9 n\left(E\left\|X_{0}\right\|^{4}\right)^{3 / 4}\left(E\left\|Y_{0}\right\|^{4}\right)^{3 / 4} L_{\mathbf{X}}(0) L_{\mathbf{Y}}(h) .
$$

Next we develop bounds for the sum when the indices are in $R_{1,3}$. We define the random functions $X_{k, k-j}^{*}=g_{\mathbf{X}}\left(\epsilon_{k}, \epsilon_{k-1}, \ldots, \epsilon_{j+1}, \epsilon_{j}^{*}, \epsilon_{j-1}^{*} \ldots\right)$ and $X_{\ell, \ell-j}^{*}=g_{\mathbf{X}}\left(\epsilon_{\ell}, \epsilon_{\ell-1}, \ldots, \epsilon_{j+1}, \epsilon_{j}^{*}\right.$, $\left.\epsilon_{j-1}^{*}, \ldots\right)$, where $\epsilon_{i}^{*},-\infty<i<\infty$ are iid copies of $\epsilon_{0}$, independent of $\epsilon_{i},-\infty<i<\infty$. It 
is clear that $\left(X_{k}, X_{\ell}\right)$ and $\left(X_{k, k-j}^{*}, X_{\ell, \ell-j}^{*}\right)$ have the same distribution and are independent of $\left(X_{0}, X_{j}\right)$. (Note that $X_{k, k-j}^{*}$ and $X_{\ell, \ell-j}^{*}$ are defined by using the same $\epsilon_{j}^{*}$ 's, making them different from $X_{k, k-j}$ and $X_{\ell, \ell-j}$ of (3.2)). By Assumption 1.2 and the Cauchy-Schwarz inequality we have

$$
\begin{aligned}
& \left|E \eta_{0, j} \eta_{k, \ell}-a(j) a(\ell-k)\right| \\
& \quad \leq \iint E\left|X_{0}(t) X_{j}(s)\left(X_{k}(s) X_{\ell}(s)-X_{k, k-j}^{*}(s) X_{\ell, \ell-j}^{*}(s)\right)\right| d t d s \\
& \quad \leq\left(E\left\|X_{0}\right\|^{4}\right)^{3 / 4}\left(\left(E\left\|X_{k}-X_{k, k-j}^{*}\right\|^{4}\right)^{1 / 4}+\left(E\left\|X_{\ell}-X_{\ell, \ell-j}^{*}\right\|^{4}\right)^{1 / 4}\right) \\
& \quad=\left(E\left\|X_{0}\right\|^{4}\right)^{3 / 4}\left(\left(E\left\|X_{0}-X_{0, k-j}\right\|^{4}\right)^{1 / 4}+\left(E\left\|X_{0}-X_{0, \ell-j}\right\|^{4}\right)^{1 / 4}\right)
\end{aligned}
$$

and similarly

$$
\begin{aligned}
& \left|E \theta_{0, j} \theta_{k+h, \ell+h}-b(j) b(\ell-k)\right| \\
& \quad \leq\left(E\left\|Y_{0}\right\|^{4}\right)^{3 / 4}\left(\left(E\left\|Y_{0}-Y_{0, k+h-j}\right\|^{4}\right)^{1 / 4}+\left(E\left\|Y_{0}-Y_{0, \ell+h-j}\right\|^{4}\right)^{1 / 4}\right) .
\end{aligned}
$$

Clearly,

$$
\sum_{(j, k, \ell) \in R_{1,3}}\left|E \eta_{0, j} \eta_{k, \ell} E \theta_{0, j} \theta_{k+h, \ell+h}-a(j) a(\ell-k) b(j) b(\ell-k)\right| \leq Q_{n, 1}+Q_{n, 2}+Q_{n, 3}
$$

with

$$
\begin{gathered}
Q_{n, 1}=\sum_{(j, k, \ell) \in R_{1,3}}\left|E \eta_{0, j} \eta_{k, \ell}-a(j) a(\ell-k)\right||b(j) b(\ell-k)|, \\
Q_{n, 2}=\sum_{(j, k, \ell) \in R_{1,3}}\left|E \theta_{0, j} \theta_{k+h, \ell+h}-b(j) b(\ell-k)\right||a(j) a(\ell-k)| \\
Q_{n, 3}=\sum_{(j, k, \ell) \in R_{1,3}}\left|E \eta_{0, j} \eta_{k, \ell}-a(j) a(\ell-k)\right|\left|E \theta_{0, j} \theta_{k+h, \ell+h}-b(j) b(\ell-k)\right| .
\end{gathered}
$$

Applying (A.2) and (A.9) we get that

$$
\begin{gathered}
Q_{n, 1} \leq\left(E\left\|X_{0}\right\|^{4}\right)^{1 / 4} E\left\|Y_{0}\right\|^{2}\left(\sum_{(j, k, \ell) \in R_{1,3}}\left(E\left\|X_{0}-X_{0, k-j}\right\|^{4}\right)^{1 / 4}\left(E\left\|Y_{0}-Y_{0, j}\right\|^{2} E\left\|Y_{0}-Y_{0, \ell-k}\right\|^{2}\right)^{1 / 2}\right. \\
\left.+\sum_{(j, k, \ell) \in R_{1,3}}\left(E\left\|X_{0}-X_{0, \ell-j}\right\|^{4}\right)^{1 / 4}\left(E\left\|Y_{0}-Y_{0, j}\right\|^{2} E\left\|Y_{0}-Y_{0, \ell-k}\right\|^{2}\right)^{1 / 2}\right) .
\end{gathered}
$$

Using the definition of $R_{1,3}$ we conclude

$$
\begin{aligned}
& \sum_{(j, k, \ell) \in R_{1,3}}\left(E\left\|X_{0}-X_{0, k-j}\right\|^{4}\right)^{1 / 4}\left(E\left\|Y_{0}-Y_{0, j}\right\|^{2} E\left\|Y_{0}-Y_{0, \ell-k}\right\|^{2}\right)^{1 / 2} \\
= & \sum_{j=1}^{h}\left(E\left\|Y_{0}-Y_{0, j}\right\|^{2}\right)^{1 / 2} \sum_{k=j}^{n}\left(\left\|X_{0}-X_{0, k-j}\right\|^{4}\right)^{1 / 4} \sum_{\ell=k}^{k+h}\left(E\left\|Y_{0}-Y_{0, \ell-k}\right\|^{2}\right)^{1 / 2} \\
\leq & h^{2} \max _{1 \leq r \leq h} E\left\|Y_{0}-Y_{0, r}\right\|^{2} J_{\mathbf{X}}(0) \\
\leq & 2 h^{2} E\left\|Y_{0}\right\|^{2} J_{\mathbf{X}}(0) .
\end{aligned}
$$


It follows similarly

$$
\sum_{(j, k, \ell) \in R_{1,3}}\left(E\left\|X_{0}-X_{0, \ell-j}\right\|^{4}\right)^{1 / 4}\left(E\left\|Y_{0}-Y_{0, j}\right\|^{2} E\left\|Y_{0}-Y_{0, \ell-k}\right\|^{2}\right)^{1 / 2} \leq 2 h^{2} E\left\|Y_{0}\right\|^{2} J_{\mathbf{X}}(0)
$$

implying

$$
Q_{n, 1} \leq 4 h^{2}\left(E\left\|X_{0}\right\|^{4}\right)^{1 / 4}\left(E\left\|Y_{0}\right\|^{2}\right)^{2} J_{\mathbf{X}}(0) .
$$

Using nearly identical arguments we obtain that

$$
Q_{n, 2} \leq 4 h^{2}\left(E\left\|Y_{0}\right\|^{4}\right)^{1 / 4}\left(E\left\|X_{0}\right\|^{2}\right)^{2} J_{\mathbf{Y}}(0)
$$

and

$$
Q_{n, 3} \leq 8 h^{2}\left(E\left\|X_{0}\right\|^{4}\right)^{3 / 4} E\left\|Y_{0}\right\|^{4} J_{\mathbf{X}}(0) .
$$

Case II. Let $R_{2}=\{(j, k, \ell): 0 \leq k \leq \ell \leq j\}, R_{2,1}=R_{2,1}(h)=\left\{(j, k, \ell) \in R_{2}: 0 \leq\right.$ $k+h \leq \ell+h \leq j\}, R_{2,2}=R_{2,2}(h)=\left\{(j, k, \ell) \in R_{2}: 0 \leq k+h \leq j \leq \ell+h\right\}$ and $R_{2,3}=R_{2,3}(h)=\left\{(j, k, \ell) \in R_{2}: 0 \leq j \leq k+h \leq \ell+h\right\}$. For $(j, k, \ell) \in R_{2,1}$ we write with the help of Assumption 1.2

$$
\begin{aligned}
\left|E \eta_{0, j} \eta_{k, \ell}\right| & =\left|E \iint X_{0}(t) X_{k}(s) X_{\ell}(s) X_{j}(t) d t d s\right| \\
& =\left|E \iint X_{0}(t) X_{k}(s) X_{\ell}(s)\left(X_{j}(t)-X_{j, j-\ell}(t) d t d s\right)\right| \\
& \leq\left(E\left\|X_{0}\right\|^{4}\right)^{3 / 4}\left(E\left\|X_{0}-X_{0, j-\ell}\right\|^{4}\right)^{1 / 4}
\end{aligned}
$$

and similarly

$$
\left|E \theta_{0, j} \theta_{k+h, \ell+h}\right| \leq\left(E\left\|Y_{0}\right\|^{4}\right)^{3 / 4}\left(E\left\|Y_{0}-Y_{0, k+h}\right\|^{4}\right)^{1 / 4}
$$

Thus we get

$$
\sum_{(j, k, \ell) \in R_{2,1}}\left|E \eta_{0, j} \eta_{k, \ell} E \theta_{0, j} \theta_{k+h} \theta_{\ell+h}\right| \leq n\left(E\left\|X_{0}\right\|^{4} E\left\|Y_{0}\right\|^{4}\right)^{3 / 4} J_{\mathbf{X}}(h) J_{\mathbf{Y}}(h) .
$$

For $(j, k, \ell) \in R_{2,2}$ similar arguments yield

$$
\sum_{(j, k, \ell) \in R_{2,2}}\left|E \eta_{0, j} \eta_{k, \ell} E \theta_{0, j} \theta_{k+h} \theta_{\ell+h}\right| \leq n\left(E\left\|X_{0}\right\|^{4} E\left\|Y_{0}\right\|^{4}\right)^{3 / 4} J_{\mathbf{X}}(h) J_{\mathbf{Y}}(h) .
$$

If $(j, k, \ell) \in R_{2,3}$, then we get

$$
\begin{aligned}
\left|E \eta_{0, j} \eta_{k, \ell}\right| & =\left|\iint X_{0}(t)\left(X_{k}(s) X_{\ell}(s) X_{j}(t)-X_{k, k}(s) X_{\ell, k}(s) X_{j, k}(t)\right) d t d s\right| \\
& \leq 3\left(E\left\|X_{0}\right\|^{4}\right)^{3 / 4}\left(E\left\|X_{0}-X_{0, k}\right\|^{4}\right)^{1 / 4}
\end{aligned}
$$

and

$$
\left|E \theta_{0, j} \theta_{k+h, \ell+h}\right| \leq 3\left(E\left\|Y_{0}\right\|^{4}\right)^{3 / 4}\left(E\left\|Y_{0}-Y_{0, j}\right\|^{4}\right)^{1 / 4}
$$

resulting in

$$
\begin{aligned}
& \sum_{(j, k, \ell) \in R_{2,3}}\left|E \eta_{0, j} \eta_{k, \ell} E \theta_{0, j} \theta_{k+h, \ell+h}\right| \\
& \quad \leq 9\left(E\left\|X_{0}\right\|^{4} E\left\|Y_{0}\right\|^{4}\right)^{3 / 4} \sum_{(j, k, \ell) \in R_{2,3}}\left(E\left\|X_{0}-X_{0, k}\right\|^{4}\right)^{1 / 4}\left(E\left\|Y_{0}-Y_{0, j}\right\|^{4}\right)^{1 / 4}
\end{aligned}
$$




$$
\leq 9 n\left(E\left\|X_{0}\right\|^{4} E\left\|Y_{0}\right\|^{4}\right)^{3 / 4} J_{\mathbf{X}}(0) J_{\mathbf{Y}}(0) \frac{h}{n}
$$

since due to the restriction $j-h \leq \ell \leq j$ on $R_{2,3}$, there are only $h$ choices for $\ell$ for any $j$. Next we consider the sum of the expected values assuming that $(j, k, \ell) \in R_{2}$. Using (A.1) and (A.2) we conclude

$$
\begin{aligned}
& \sum_{(j, k, \ell) \in R_{2}}|a(j) a(\ell-k) b(j) b(\ell-k)| \\
& \leq E\left\|X_{0}\right\|^{2} E\left\|Y_{0}\right\|^{2} \sum_{(j, k, \ell) \in R_{2}}\left(E\left\|X_{0}-X_{0, j}\right\|^{2} E\left\|X_{0}-X_{0, \ell-k}\right\|^{2} E\left\|Y_{0}-Y_{0, j}\right\|^{2} E\left\|Y_{0}-Y_{0, \ell-k}\right\|^{2}\right)^{1 / 2}
\end{aligned}
$$

and

$$
\begin{aligned}
& \sum_{(j, k, \ell) \in R_{2}}\left(E\left\|X_{0}-X_{0, j}\right\|^{2} E\left\|X_{0}-X_{0, \ell-k}\right\|^{2} E\left\|Y_{0}-Y_{0, j}\right\|^{2} E\left\|Y_{0}-Y_{0, \ell-k}\right\|^{2}\right)^{1 / 2} \\
\leq & \sum_{0 \leq k \leq \ell \leq N}\left(E\left\|X_{0}-X_{0, \ell-k}\right\|^{2} E\left\|Y_{0}-Y_{0, \ell-k}\right\|^{2}\right)^{1 / 2}\left(\sum_{j \geq \ell}\left(E\left\|X_{0}-X_{0, j}\right\|^{2} E\left\|Y_{0}-Y_{0, j}\right\|^{2}\right)^{1 / 2}\right) \\
= & \sum_{\ell=0}^{N}\left(\sum_{m=0}^{\ell}\left(E\left\|X_{0}-X_{0, m}\right\|^{2} E\left\|Y_{0}-Y_{0, m}\right\|^{2}\right)^{1 / 2}\right)\left(\sum_{j \geq \ell}\left(E\left\|X_{0}-X_{0, j}\right\|^{2} E\left\|Y_{0}-Y_{0, j}\right\|^{2}\right)^{1 / 2}\right) \\
\leq & \sum_{m=0}^{\infty}\left(E\left\|X_{0}-X_{0, m}\right\|^{2} E\left\|Y_{0}-Y_{0, m}\right\|^{2}\right)^{1 / 2} \sum_{\ell=0}^{\infty} \sum_{j=\ell}^{\infty}\left(E\left\|X_{0}-X_{0, j}\right\|^{2} E\left\|Y_{0}-Y_{0, j}\right\|^{2}\right)^{1 / 2} \\
\leq & 2\left(E\left\|Y_{0}\right\|^{2}\right)^{1 / 2} L_{\mathbf{X}}(0) \sum_{\ell=0}^{\infty} \ell\left(E\left\|X_{0}-X_{0, \ell}\right\|^{2} E\left\|Y_{0}-Y_{0, \ell}\right\|^{2}\right)^{1 / 2} \\
\leq & 4 E\left\|Y_{0}\right\|^{2} L_{\mathbf{X}}(0) \sum_{\ell=0}^{\infty} \ell\left(E\left\|X_{0}-X_{0, \ell}\right\|^{2}\right)^{1 / 2}
\end{aligned}
$$

by Abel's summation formula. Finally we consider the case when the summation is over $R_{3}=\{0 \leq k \leq j \leq \ell \leq n-1\}$. Let $R_{3,1}=\left\{(j, k, \ell) \in R_{3}: 0 \leq k+h \leq j \leq \ell+h\right\}$ and $R_{3,2}=\left\{(j, k, \ell) \in R_{3}: 0 \leq j \leq k+h \leq \ell+h\right\}$. If $(j, k, \ell) \in R_{3,1}$ we have

$$
\begin{aligned}
\left|E \eta_{0, j} \eta_{k, \ell}\right| & =\left|E \iint X_{0}(t) X_{k}(s) X_{j}(t) X_{\ell}(s) d t d s\right| \\
& =E \iint\left|X_{0}(t) X_{k}(s) X_{j}(t)\left(X_{\ell}(s)-X_{\ell, \ell-j}(s)\right)\right| d t d s \\
& \leq\left(E\left\|X_{0}\right\|^{4}\right)^{3 / 4}\left(E\left\|X_{0}-X_{0, \ell-j}\right\|^{4}\right)^{1 / 4}
\end{aligned}
$$

and similarly

$$
\begin{aligned}
\left|E \theta_{0, j} \theta_{k+h, \ell+h}\right| & =\left|E \iint Y_{0}(t)\left(Y_{k+h}(s) Y_{j}(t) Y_{\ell+h}(s)-Y_{k+h, k+h}(s) Y_{j, k+h}(t) Y_{\ell+h, k+h}(s)\right) d t d s\right| \\
& \leq 3\left(E\left\|Y_{0}\right\|^{4}\right)^{3 / 4}\left(E\left\|Y_{0}-Y_{0, k+h}\right\|^{4}\right)^{1 / 4}
\end{aligned}
$$

resulting in

$$
\frac{1}{n} \sum_{(j, k, \ell) \in R_{3,1}}\left|E \eta_{0, j} \eta_{k, \ell} E \theta_{k+h} \theta_{\ell+h}\right| \leq 3\left(E\left\|X_{0}\right\|^{4} E\left\|X_{0}\right\|^{4}\right)^{3 / 4} J_{\mathbf{X}}(0) J_{\mathbf{Y}}(h) .
$$


In case of $(j, k, \ell) \in R_{3,2}$ the upper bound in (A.10) still holds but for the $\theta$ 's we have

$$
\begin{aligned}
\left|E \theta_{0, j} \theta_{k+h, \ell+h}\right| & =\left|E \iint Y_{0}(t)\left(Y_{j}(t) Y_{k+h}(s) Y_{\ell+h}(s)-Y_{j, j}(t) Y_{k+h, j}(s) Y_{\ell+h, j}(s)\right) d t d s\right| \\
& \leq 3\left(E\left\|Y_{0}\right\|^{4}\right)^{3 / 4}\left(E\left\|Y_{0}-Y_{0, j}\right\|^{4}\right)^{1 / 4} .
\end{aligned}
$$

Since for all $(j, k, \ell) \in R_{3,2}$ we have that $j-h \leq k \leq j$ we conclude

$$
\begin{aligned}
& \sum_{(j, k, \ell) \in R_{3,2}}\left|E \eta_{0, j} \eta_{k+h} E \theta_{0, j} \theta_{k+h, \ell+h}\right| \\
& \leq 3\left(E\left\|X_{0}\right\|^{4} E\left\|Y_{0}\right\|^{4}\right)^{3 / 4} \sum_{(j, k, \ell) \in R_{3,2}}\left(E\left\|X_{0}-X_{0, \ell-j}\right\|^{4} E\left\|Y_{0}-Y_{0, j}\right\|^{4}\right)^{1 / 4} \\
& \leq 3 h\left(E\left\|X_{0}\right\|^{4} E\left\|Y_{0}\right\|^{4}\right)^{3 / 4} \sum_{(j, k, \ell) \in R_{3,2}} \sum_{0 \leq j \leq \ell \leq n}\left(E\left\|X_{0}-X_{0, \ell-j}\right\|^{4} E\left\|Y_{0}-Y_{0, j}\right\|^{4}\right)^{1 / 4} \\
& \leq 3 h\left(E\left\|X_{0}\right\|^{4} E\left\|Y_{0}\right\|^{4}\right)^{3 / 4} J_{\mathbf{X}}(0) J_{\mathbf{Y}}(0) .
\end{aligned}
$$

Furthermore, by (A.1) and (A.2) we get

$$
\begin{aligned}
& \sum_{(j, k, \ell) \in R_{3}}|a(j) a(\ell-k) b(j) b(\ell-k)| \\
& \leq E\left\|X_{0}\right\|^{2} E\left\|Y_{0}\right\|^{2} \sum_{0 \leq k \leq j \leq \ell}\left(E\left\|X_{0}-X_{0, j}\right\|^{2} E\left\|X_{0}-X_{0, \ell-k}\right\|^{2} E\left\|Y_{0}-Y_{0, j}\right\|^{2} E\left\|Y_{0}-Y_{0, \ell-k}\right\|^{2}\right)^{1 / 2} \\
& \leq E\left\|X_{0}\right\|^{2} E\left\|Y_{0}\right\|^{2} \sum_{0 \leq k \leq \ell}\left(E\left\|X_{0}-X_{0, \ell-k}\right\|^{2} E\left\|Y_{0}-Y_{0, \ell-k}\right\|^{2}\right)^{1 / 2} \\
& \quad \times \sum_{j=k}^{\infty}\left(E\left\|X_{0}-X_{0, j}\right\|^{2} E\left\|Y_{0}-Y_{0, j}\right\|^{2}\right)^{1 / 2} \\
& \leq 2 E\left\|X_{0}\right\|^{2} E\left\|Y_{0}\right\|^{3} L_{\mathbf{X}}(0) \sum_{k=0}^{\infty} \sum_{j=k}^{\infty}\left(E\left\|X_{0}-X_{0, j}\right\|^{2} E\left\|Y_{0}-Y_{0, j}\right\|^{2}\right)^{1 / 2} \\
& \leq 4 E\left\|X_{0}\right\|^{3} E\left\|Y_{0}\right\|^{3} L_{\mathbf{X}}(0) \sum_{k=0}^{\infty} k\left(E\left\|Y_{0}-Y_{0, k}\right\|^{2}\right)^{1 / 2} .
\end{aligned}
$$

This completes the proof of the lemma.

\section{Appendix B. Asymptotics for means and variances of $\tilde{T}_{n, H}$ And $\bar{T}_{n, H, m}$} We recall that $\bar{T}_{n, H, m}$ is determined by the $m$-dependent random functions $\left\{X_{i, m},-\infty<\right.$ $i<\infty\}$ and $\left\{Y_{i, m},-\infty<i<\infty\right\}$ defined in (3.2) and (3.3), respectively.

Lemma B.1. If Assumptions 1.1-1.5 and (3.1) hold, then for every $m \geq 1$ we have that

$$
\begin{gathered}
\frac{E \bar{T}_{n, H, m}}{2 H+1}=\bar{\mu}(m)+O(1 / n), \\
\bar{\mu}(m)=\sum_{\ell=-m}^{m} \int E\left[X_{0, m}(t) X_{\ell, m}(t)\right] d t \int E\left[Y_{0, m}(s) Y_{\ell, m}(s)\right] d s,
\end{gathered}
$$




$$
\frac{E \tilde{T}_{n, H}}{2 H+1}=\mu+O(1 / n),
$$

where $\mu$ is defined in (1.4), and

$$
\begin{gathered}
\lim _{n \rightarrow \infty} \frac{\operatorname{var}\left(\bar{T}_{n, H, m}\right)}{2 H+1}=\bar{\sigma}^{2}(m), \\
\bar{\sigma}^{2}(m)=\sum_{h=-m}^{m} \bar{\sigma}_{h}^{2}(m), \\
\bar{\sigma}_{h}^{2}(m)=2 \int \cdots \int\left(\sum_{\ell=-m}^{m} E\left[X_{0, m}(t) X_{\ell, m}(s)\right] E\left[Y_{0, m}(u) Y_{\ell+h, m}(v)\right]\right)^{2} d t d s d u d v, \\
\lim _{m \rightarrow \infty} \bar{\sigma}^{2}(m)=\sigma^{2},
\end{gathered}
$$

where $\sigma^{2}$ is defined in (1.5).

Proof. First we note that by the stationarity of $X_{i, m}$ and $Y_{j, m}$ we have

$$
\begin{aligned}
\lim _{n \rightarrow \infty} \frac{E \bar{T}_{n, H, M}}{2 H+1}= & n E \int \bar{C}_{n, 0, m}^{2}(t, s) d t d s \\
= & \frac{1}{n} \sum_{1 \leq i, j \leq n} \iint E\left[X_{i, m}(t) X_{j, m}(t)\right] E\left[Y_{i+h, m}(s) Y_{j+h, m}(s)\right] d t d s \\
= & \int E X_{0, m}(t) X_{0, m}(t) d t \int E Y_{0, m}(t) Y_{0, m}(t) d s \\
& +2 \sum_{1 \leq \ell \leq n-1}\left(1-\frac{\ell}{n}\right) \iint E\left[X_{0, m}(t) X_{\ell, m}(t)\right] E\left[Y_{0, m}(s) Y_{\ell, m}(s)\right] d t d s \\
= & \bar{\mu}(m)+O(1 / n),
\end{aligned}
$$

proving B.1. Similarly,

$$
\begin{aligned}
n E \tilde{\xi}_{n, h} & =\frac{1}{n} \sum_{1 \leq i, j \leq n} \iint E\left[X_{i}(t) X_{j}(t)\right] E\left[Y_{i+h}(s) Y_{j+h}(s)\right] d t d s \\
& =\int E X_{0}(t) X_{0}(t) d t \int E Y_{0}(t) Y_{0}(t) d s \\
& +2 \sum_{1 \leq \ell \leq n-1}\left(1-\frac{\ell}{n}\right) \iint E\left[X_{0}(t) X_{\ell}(t)\right] E\left[Y_{0}(s) Y_{\ell}(s)\right] d t d s
\end{aligned}
$$

and therefore by Assumptions 1.2 and 1.3 we have (B.2).

To prove (B.3) we note that by stationarity we have

$$
\begin{aligned}
\operatorname{var}\left(\bar{T}_{n, H, m}\right) & =\sum_{h, h^{\prime}=-H}^{H} \operatorname{cov}\left(\bar{\xi}_{n, h, m}, \bar{\xi}_{n, h^{\prime}, m}\right) \\
& =(2 H+1) \sum_{h=-2 H}^{2 H} \operatorname{cov}\left(\bar{\xi}_{n, 0, m}, \bar{\xi}_{n, h, m}\right)-\sum_{h=-2 H}^{2 H}|h| \operatorname{cov}\left(\bar{\xi}_{n, 0, m}, \bar{\xi}_{n, h, m}\right)
\end{aligned}
$$




$$
=(2 H+1) \sum_{h=-2 H}^{2 H} \operatorname{cov}\left(\bar{\xi}_{n, 0, m}, \bar{\xi}_{n, h, m}\right)+O(1),
$$

since by the arguments used in Section A

$$
\sum_{-2 H \leq h \leq 2 H}|h|\left|\operatorname{cov}\left(\bar{\xi}_{n, 0, m}, \bar{\xi}_{n, h, m}\right)\right|=O(1) .
$$

Next we show that

$$
\max _{-2 H \leq h \leq 2 H}\left|\operatorname{cov}\left(\bar{\xi}_{n, 0, m}, \bar{\xi}_{n, h, m}\right)-\bar{\sigma}_{h}^{2}(m)\right|=O(H / n) .
$$

Using the definition of $\bar{\xi}_{n, h, m}$ we have

$$
\operatorname{cov}\left(\bar{\xi}_{n, 0, m}, \bar{\xi}_{n, h, m}\right)=\frac{1}{n^{2}} \sum_{1 \leq i, j, k, \ell \leq n} \chi_{i, j, k, \ell}(h),
$$

where $\chi_{i, j, k, \ell}(h)=\chi_{i, j, k, \ell}^{(1)}(h)-\chi_{i, j, k, \ell}^{(2)}(h)$,

$$
\begin{aligned}
& \chi_{i, j, k, \ell}^{(1)}(h) \\
& =\int \cdots \int E\left[X_{i, m}(t) X_{j, m}(t) X_{k, m}(u) X_{\ell, m}(u)\right] E\left[Y_{i, m}(s) Y_{j, m}(s) Y_{k+h, m}(v) Y_{\ell+h, m}(v)\right] d t d s d u d v
\end{aligned}
$$

and

$$
\begin{aligned}
& \chi_{i, j, k, \ell}^{(2)}(h) \\
& =\int \cdots \int E\left[X_{i, m}(t) X_{j, m}(t)\right] E\left[Y_{i, m}(s) Y_{j, m}(s)\right] E\left[X_{i, m}(u) X_{j, m}(u)\right] E\left[Y_{i, m}(v) Y_{j, m}(v)\right] d t d s d u d v .
\end{aligned}
$$

Let $R_{1}=\{(i, j, k, \ell): 1 \leq i, j \leq k, \ell \leq n\}$ and $R_{2}=\{(i, j, k, \ell): 1 \leq i, k \leq j, \ell \leq n\}$. Using stationarity we get

$$
\sum_{1 \leq i, j, k, \ell \leq n} \chi_{i, j, k, \ell}(h)=2 \sum_{(i, j, k, \ell) \in R_{1}} \chi_{i, j, k, \ell}(h)+4 \sum_{(i, j, k, \ell) \in R_{2}} \chi_{i, j, k, \ell}(h) .
$$

By the $m$-dependence of $X_{i, m}$ and $Y_{i, m}$ we obtain that

$$
\max _{|h| \leq 2 H} \frac{1}{n^{2}}\left|\sum_{(i, j, k, \ell) \in R_{1}} \chi_{i, j, k, \ell}(h)\right|=O\left(\frac{1}{n}\right) .
$$

Also, it is easy to check that

$$
\max _{|h| \leq 2 H} \frac{1}{n^{2}}\left|\sum_{(i, j, k, \ell) \in R_{2}} \chi_{i, j, k, \ell}^{(2)}(h)\right|=O\left(\frac{1}{n}\right)
$$

and therefore we need to study $\sum_{(i, j, k, \ell) \in R_{2}} \chi_{i, j, k, \ell}^{(1)}(h)$ only. We decompose $R_{2}$ as $R_{2}=$ $\cup_{i=1}^{4} R_{2, i}$, where

$$
\begin{aligned}
& R_{2,1}=\{(i, j, k, \ell): 1 \leq i \leq k \leq j \leq \ell \leq n\}, \quad R_{2,2}=\{(i, j, k, \ell): 1 \leq i \leq k \leq \ell<j \leq n\} \\
& R_{2,3}=\{(i, j, k, \ell): 1 \leq k<i \leq \ell<j \leq n\}, \quad R_{2,4}=\{(i, j, k, \ell): 1 \leq k<i \leq j \leq \ell \leq n\} .
\end{aligned}
$$


Furthermore, let $R_{2,1}^{(1)}=\{(i, j, k, \ell): k \leq j \leq k+h+m\} \cap R_{2,1}$ and $R_{2,1}^{(2)}=\{(i, j, k, \ell)$ : $j>k+h+m\} \cap R_{2,1}$. Using again $m$-dependence we get that

$$
\frac{1}{n^{2}} \max _{|h| \leq 2 H}\left|\sum_{(i, j, k, \ell) \in R_{2,1}^{(1)}} \chi_{i, j, k, \ell}^{(1)}(h)\right|=O\left(\frac{H}{n}\right) .
$$

Furthermore,

$$
\begin{aligned}
& \sum_{(i, j, k, \ell) \in R_{2,1}^{(2)}} \chi_{i, j, k, \ell}^{(1)}(h) \\
& =\int \ldots / \sum_{i=1}^{n} \sum_{k=i}^{n} \sum_{j=k+m+h}^{n} \sum_{\ell=j}^{n} E\left[X_{i, m}(t) X_{j, m}(t) X_{k, m}(u) X_{\ell, m}(u)\right] \\
& \quad \times E\left[Y_{i, m}(s) Y_{j, m}(s) Y_{k+h, m}(v) Y_{\ell+h, m}(v)\right] d t d s d u d v \\
& =\int \ldots / \sum_{i=1}^{n} \sum_{k=i}^{n} \sum_{j=k+m+h}^{n} \sum_{\ell=j}^{n} E\left[X_{i, m}(t) X_{k, m}(u)\right] E\left[X_{j, m}(t) X_{\ell, m}(u)\right] \\
& \quad \times E\left[Y_{i, m}(s) Y_{k+h, m}(v)\right] E\left[Y_{j, m}(s) Y_{\ell+h, m}(v)\right] d t d s d u d v \\
& =\int \ldots / \sum_{i=1}^{n} \sum_{r=0}^{n-i} \sum_{j=r+i+m+h}^{n} \sum_{p=0}^{n-j} E\left[X_{0, m}(t) X_{r, m}(u)\right] E\left[X_{0, m}(t) X_{p, m}(u)\right] \\
& \quad \times E\left[Y_{0, m}(s) Y_{r+h, m}(v)\right] E\left[Y_{0, m}(s) Y_{p+h, m}(v)\right] d t d s d u d v \\
& =U_{n, 1}(h)+\int \ldots \int \sum_{i=1}^{n-m} \sum_{r=0}^{m} \sum_{j=i+m+h}^{n-m} \sum_{p=0}^{m} E\left[X_{0, m}(t) X_{r, m}(u)\right] E\left[X_{0, m}(t) X_{p, m}(u)\right] \\
& \quad \times E\left[Y_{0, m}(s) Y_{r+h, m}(v)\right] E\left[Y_{0, m}(s) Y_{p+h, m}(v)\right] d t d s d u d v
\end{aligned}
$$

and

$$
\max _{|h| \leq 2 H}\left|U_{n, 1}(h)\right|=O(n) .
$$

Elementary algebra and $H / n \rightarrow 0$ give

$$
\sup _{|h| \leq 2 H}\left|\sum_{i=1}^{n-m} \sum_{j=i+m+h}^{n-m} 1-\frac{n^{2}}{2}\right|=O(n)
$$

and therefore

$$
\begin{aligned}
& \int \ldots \int \sum_{i=1}^{n-m} \sum_{r=0}^{m} \sum_{j=i+m+h}^{n-m} \sum_{p=0}^{m} E\left[X_{0, m}(t) X_{r, m}(u)\right] E\left[X_{0, m}(t) X_{p, m}(u)\right] \\
& \quad \times E\left[Y_{0, m}(s) Y_{r+h, m}(v)\right] E\left[Y_{0, m}(s) Y_{p+h, m}(v)\right] d t d s d u d v \\
& =\frac{n^{2}}{4} \bar{\sigma}_{h, 1}^{2}(m)+U_{n, 2}(h)
\end{aligned}
$$

where

$$
\bar{\sigma}_{h, 1}^{2}(m)=2 \int \cdots \int \sum_{r=0}^{m} \sum_{p=0}^{m} E\left[X_{0, m}(t) X_{r, m}(u)\right] E\left[X_{0, m}(t) X_{p, m}(u)\right]
$$




$$
\times E\left[Y_{0, m}(s) Y_{r+h, m}(v)\right] E\left[Y_{0, m}(s) Y_{p+h, m}(v)\right] d t d s d u d v
$$

and

$$
\max _{|h| \leq 2 H}\left|U_{n, 2}(h)\right|=O(n)
$$

Thus we have

$$
\max _{|h| \leq 2 H}\left|\frac{4}{n^{2}} \sum_{(i, j, k, \ell) \in R_{2,1}} \chi_{i, j, k, \ell}^{(1)}(h)-\bar{\sigma}_{h, 1}^{2}(m)\right|=O\left(\frac{H}{n}\right) .
$$

In a similar fashion it can be shown that

$$
\max _{|h| \leq 2 H}\left|\frac{4}{n^{2}} \sum_{(i, j, k, \ell) \in R_{2, q}} \chi_{i, j, k, \ell}^{(1)}(h)-\bar{\sigma}_{h, q}^{2}(m)\right|=O\left(\frac{H}{n}\right), \quad q=2,3,4,
$$

where

$$
\begin{aligned}
\bar{\sigma}_{h, 2}^{2}(m)=2 \int & \ldots \int \sum_{r=0}^{m} \sum_{p=-m}^{-1} E\left[X_{0, m}(t) X_{r, m}(u)\right] E\left[X_{0, m}(t) X_{p, m}(u)\right] \\
& \times E\left[Y_{0, m}(s) Y_{r+h, m}(v)\right] E\left[Y_{0, m}(s) Y_{p+h, m}(v)\right] d t d s d u d v \\
\bar{\sigma}_{h, 3}^{2}(m)=2 \int & \cdots \int \sum_{r=-m}^{-1} \sum_{p=-m}^{-1} E\left[X_{0, m}(t) X_{r, m}(u)\right] E\left[X_{0, m}(t) X_{p, m}(u)\right] \\
& \times E\left[Y_{0, m}(s) Y_{r+h, m}(v)\right] E\left[Y_{0, m}(s) Y_{p+h, m}(v)\right] d t d s d u d v \\
\bar{\sigma}_{h, 4}^{2}(m)=2 \int & \cdots \int \sum_{r=-m}^{-1} \sum_{p=0}^{m} E\left[X_{0, m}(t) X_{r, m}(u)\right] E\left[X_{0, m}(t) X_{p, m}(u)\right] \\
& \times E\left[Y_{0, m}(s) Y_{r+h, m}(v)\right] E\left[Y_{0, m}(s) Y_{p+h, m}(v)\right] d t d s d u d v .
\end{aligned}
$$

Since $\bar{\sigma}_{h}^{2}(m)=\bar{\sigma}_{1,4}^{2}(m)+\cdots+\bar{\sigma}_{h, 4}^{2}(m)$, the proof of (B.5) is complete.

Observing that $\bar{\sigma}_{h}^{2}(m)=0$, if $|h|>2 m+1$, and $H / n \rightarrow 0$, (B.3) is established.

The series in the definitions of $\sigma^{2}$ are absolutely convergent and Assumptions 1.2 and 1.3 imply that $\bar{\sigma}_{h}^{2}(m) \rightarrow \sigma_{h}^{2}$, as $m \rightarrow \infty$, proving (B.4).

Lajos Horváth, Department of Mathematics, University of Utah, Salt Lake City, Ut 84112-0090 USA

Gregory Rice, Department of Mathematics, University of Utah, Salt Lake City, Ut 84112-0090 USA 